Manuscript \#: aac4354_v1

\title{
In vivo aspects of protein folding and quality control
}

\author{
David Balchin, Manajit Hayer-Hartl and F. Ulrich Hartl* \\ Department of Cellular Biochemistry, Max Planck Institute of Biochemistry, Am Klopferspitz 18, 82152 \\ Martinsried, Germany. \\ *To whom correspondence should be addressed. E-mail: uhartl@biochem.mpg.de
}

Short Title: Protein folding and quality control 


\begin{abstract}
Most proteins must fold into unique three-dimensional structures to perform their biological functions. In the crowded cellular environment, newly-synthesized proteins are at risk of misfolding and forming toxic aggregate species. To ensure efficient folding, different classes of molecular chaperones receive the nascent protein chain emerging from the ribosome and guide it along a productive folding pathway. Because proteins are structurally dynamic, constant surveillance of the proteome by an integrated network of chaperones and protein degradation machineries is required to maintain protein homeostasis (proteostasis). The capacity of this proteostasis network declines during aging, facilitating neurodegeneration and other chronic diseases associated with protein aggregation. Understanding the proteostasis network holds the promise of identifying targets for pharmacological intervention in these pathologies.
\end{abstract}


Proteins are involved in almost every biological process. A typical mammalian cell expresses 1020,000 different proteins, which are synthesized on ribosomes as linear chains up to several thousand amino acids (aa) in length. To function, most newly-synthesized proteins must fold into a defined three-dimensional structure. How this is accomplished and how cells maintain the integrity of their proteome (proteostasis (1)) in a range of external and endogenous stress conditions is a problem of paramount biological and medical relevance.

The folded structures of proteins are only marginally stable, and subtle changes due to mutation may tip the balance (2). Furthermore, a substantial fraction of proteins (15-30\% of mammalian proteomes) lack ordered structure partially or entirely (3), and the formation of toxic aggregates by such metastable proteins is associated with Alzheimer's and Parkinson's disease. Thus, protein quality control and the maintenance of proteome balance are critical for cellular and organismal health. To ensure proteostasis, organisms from all domains of life invest in an extensive quality control network, integrating molecular chaperones, which mediate protein folding and conformational repair, with the ubiquitin-proteasome system (UPS) and autophagy, which remove terminally misfolded proteins and aggregates. Importantly, the capacity of the proteostasis network $(\mathrm{PN})$ declines during aging (4), facilitating the emergence of chronic diseases caused by protein aggregation, including neurodegeneration, type II diabetes, heart disease and certain forms of cancer.

Here we review advances made over the last 15 years in understanding how cells support protein folding and maintain proteostasis. We focus on the mechanisms of molecular chaperones in the cytosol, and the ways that chaperone systems cooperate to form a functional PN. In particular, we highlight the differences in the pathways and energy landscapes of protein folding 
in vivo and in vitro. For a detailed discussion of the protein folding machineries of the endoplasmic reticulum, see (5).

\section{Molecular Chaperones - Machineries of Protein Folding}

Fundamental insight into protein folding was provided by Anfinsen's pioneering experiments in the 1950s, which showed that small proteins refold spontaneously in vitro on removal from denaturant (6). This finding demonstrated the primacy of the amino acid sequence in determining a protein's native conformation, and also implied that folding occurs without the aid of additional factors. However, evidence began to emerge in the 1980s that certain proteins require assistance by molecular chaperones for folding in vivo (7). Research over the last two decades has firmly established the essential role of chaperone machineries in allowing newly-synthesized proteins to fold efficiently and at a biologically relevant time-scale.

While small proteins up to $\sim 100$ aa may fold rapidly (within milliseconds) and with full yield in vitro (8), folding is often inefficient for larger proteins, due to off-pathway aggregation. Proteins $>100$ aa constitute the major fraction of all proteomes, with large multidomain proteins strongly increasing in number from prokaryotes to eukaryotes (Fig. 1). The folding of such proteins in vivo is further compounded by macromolecular crowding $(300-400 \mathrm{~g} / \mathrm{L}$ total protein in the cytosol) (9), which enhances the tendency of folding intermediates and misfolded states to aggregate. A major function of the chaperone network is to prevent such aberrant interactions, which are often irreversible. We define a molecular chaperone as any protein that interacts with, stabilizes or helps another protein to acquire its functionally active conformation, without being present in its final structure (7). 


\section{Folding and Aggregation}

The folding process is inherently error-prone, owing to the large number of possible conformations a protein chain can adopt ( $>10^{30}$ for a 100 aa protein). Moreover, folding relies on the cooperation of many weak, non-covalent interactions and involves amino acid contacts both close in sequence and far apart (8). Hydrophobic forces are particularly relevant in driving chain collapse and the burial of non-polar amino acids within the core of the folding structure (in the case of soluble proteins), restricting the conformational space that must be searched during folding (10). However, the free-energy landscape towards the thermodynamically favorable native state is often rugged, which means the molecules need to cross substantial kinetic energy barriers and consequently populate folding intermediates (8) (Fig. 2A). Such kinetically trapped intermediates are the rule for larger proteins, entailing the danger of misfolding due to the formation of non-native interactions (10). Slow steps in folding that lead to the accumulation of folding intermediates include prolyl isomerization and disulfide bond formation (in secretory proteins), which can be accelerated by prolyl isomerases and protein disulfide isomerases, respectively $(5,11)$.

Partially folded or misfolded proteins typically expose hydrophobic amino acid residues and regions of unstructured polypeptide backbone to the solvent, features that can give rise to aggregation. Like folding, aggregation is largely driven by hydrophobic forces but is highly concentration dependent (12). While the majority of aggregates are amorphous, a subset of nonnative proteins aggregate to form so-called amyloid fibrils, which are structurally defined by $\beta$ strands running perpendicular to the long fibril axis (cross- $\beta$-structure) (Fig. 2A). Fibril formation is often preceded by the accumulation of oligomeric aggregates, which are thought to play key roles in disease (Fig. 2A). 
Molecular chaperones that function broadly in de novo folding include the chaperonins (Hsp60), the Hsp70 and Hsp90 systems. They recognize hydrophobic amino acid residues exposed by non-native proteins and promote folding through ATP-regulated cycles of protein binding and release. In this mechanism of kinetic partitioning, release of hydrophobic elements allows folding to proceed, while (re)binding of non-native protein blocks aggregation and may reverse misfolded states (Fig. 2B).

\section{Protein Folding in the Context of Translation}

Protein folding in vivo occurs in the context of the vectorial translation of the polypeptide chain on the ribosome from $\mathrm{N}$ - to $\mathrm{C}$-terminus, a fundamental difference from refolding of a chemically denatured protein in vitro where all elements of the polypeptide chain are simultaneously available.

Translation is slow compared to folding. For example, it takes eukaryotic ribosomes $\sim 25$ sec to synthesize a 100 aa protein ( $\sim 5 \mathrm{sec}$ for bacterial ribosomes) (13), suggesting that folding can begin cotranslationally. The nascent chain exit channel of the large ribosome subunit is $\sim 100$ $\AA$ long and for the most part 10-20 $\AA$ wide, providing just enough space for $\alpha$-helices or small tertiary structure elements to form (14) (Fig. 3). As a consequence, the C-terminal 30-40 aa of the translating chain are topologically restricted and cannot participate in the long-range interactions necessary for the cooperative folding of larger domains. The exit channel expands towards its opening, however, forming an "exit port" that may allow the emerging chain to explore local peptide conformations $(15,16)$. The channel of the bacterial ribosome was recently shown to provide sufficient space for the folding of a 29 aa zinc-binding protein (17). A limited number of small single-domain proteins ( $~ 80$ proteins $<50$ aa in E. coli; see Fig. 1) may thus fold 
to completion before emerging from the ribosome. A five-helix domain of 70 aa was observed to form a compact non-native intermediate in the exit port that rearranges into a native-like structure after the full domain sequence emerges from the ribosome (18). Moreover, the ribosome surface contributes to avoiding misfolding of incomplete chains that would aggregate in free solution $(19,20)$. These studies provide fascinating evidence that the ribosome modulates the folding pathway: Because folding is rate-limited by translation, the nascent protein is allowed to sample available conformations in a quasi-equilibrium (16), with small modules beginning to form inside the channel and coalescing upon emergence from the ribosome, perhaps reducing kinetic traps along the folding pathway. Chaperone binding to the emerging chain may further avoid misfolding, as described in the following section.

While most single-domain proteins reach their native state post-translationally once all elements of the nascent chain are available outside the ribosome $(18,20-22)$, cotranslational folding is critical for the biogenesis of many multidomain proteins. As noted previously (23), the sequential folding of domains emerging from the ribosome avoids the formation of misfolded states resulting from non-native inter-domain contacts $(24,25)$ (Fig. 3). The cotranslational formation of folded domains also allows subunit assembly to initiate on ribosome-bound polypeptides, as shown for the LuxA and LuxB subunits of bacterial luciferase (26). Such a mechanism is facilitated by the operon structure of bacterial genes where subunits of an oligomeric complex are often encoded by the same messenger RNA (mRNA). Of note, adjacent ribosomes in poly-ribosome complexes have been observed to be closely associated in a manner that maximizes the distance between their polypeptide exit sites $(27,28)$. This topology would disfavor aberrant interactions between nascent chains similar in length and folding status. Thus, a 
partially folded nascent chain may preferentially assemble with a completed subunit that has already left the ribosome.

The rate of translation can be manipulated by cells to optimize folding (29-34).

Modifying translation speed by altering codon usage affects the conformation and function of the clock protein FREQUENCY (35) and the multi-drug resistance efflux pump MDR1 (36). Thus, a nuanced picture of translation is emerging, in which the rate of protein synthesis has been tuned in evolution to optimize folding and prevent misfolding of the nascent polypeptide.

\section{Chaperone Networks in the Cytosol}

Several evolutionary conserved families of molecular chaperones guide proteins along productive folding pathways, avoiding and sometime reversing misfolding and aggregation. Their members are often referred to as stress- or heat shock proteins (Hsp) because they are upregulated in conditions of conformational stress. The major chaperone families are classified by molecular weight (Hsp40, Hsp60, Hsp70, Hsp90, Hsp100 and the small Hsp).

The organizational principles of cytosolic chaperone pathways are highly conserved. For the majority of proteins too large to fold in association with the ribosome, chaperones delay chain compaction, preventing misfolding, until sufficient structural elements are available for folding to be productive. Ribosome-binding chaperones (Trigger factor, TF, in bacteria; ribosome-associated complex, RAC, and nascent-chain-associated complex, NAC, in eukarya) interact first with the nascent polypeptide, followed by chaperones that have no direct affinity for the ribosome, including the classical Hsp70 system (DnaK/DnaJ in bacteria; Hsp70/Hsp40 in eukarya) (Fig. 4). Completion of folding may either be accomplished by Hsp70 or require transfer to the chaperonin (GroEL/ES in bacteria; TRiC in eukarya) or the Hsp90 system (HtpG in bacteria) (Fig. 4). The different chaperone machineries interact directly, or use specific 
adaptor proteins to facilitate client transfer. In this way, the folding protein is constantly

protected from aberrant interactions and premature degradation in the cytosol $(37,38)$. The ATPindependent sHsp function in buffering aggregation and cooperate with Hsp70 in folding reactions (39). In addition to these promiscuous chaperone systems, specific assembly chaperones may assist the formation of oligomeric protein complexes by interacting with their folded subunits $(40,41)$.

\section{Chaperone functions on the ribosome}

In bacteria, the abundant $\mathrm{TF}$ binds to the large ribosome subunit at the opening of the polypeptide exit tunnel and interacts with the nascent chains of most proteins $>100$ aa in an ATPindependent manner $(42,43)$. Although TF is not essential in E. coli under laboratory growth conditions, the combined deletion of TF and DnaK (the major bacterial Hsp70) is lethal above $30^{\circ} \mathrm{C}$, resulting in bulk protein aggregation $(13,43)$. TF $(\sim 50 \mathrm{kDa})$ has an elongated threedomain structure (22) and binds to hydrophobic stretches in nascent chains, thereby delaying chain collapse $(22,44-47)$. As a result, TF slows the rate of cotranslational folding but increases the yield of the folding reaction (48). For longer nascent chains, TF cooperates with the ATPregulated DnaK/DnaJ system (13) (Fig. 4A). Release from TF appears to be governed by the propensity of the bound peptide to bury hydrophobic segments as translation proceeds. A similar mechanism of folding is exemplified by the ATP-independent chaperone Spy in the bacterial periplasm, where client proteins remain associated with the chaperone until all hydrophobic elements are buried in the folded structure (49).

In eukaryotes, RAC and NAC may fulfill a role similar to $\mathrm{TF}$ in protein folding. RAC in S. cerevisiae and other fungi is a complex of the Hsp70 chaperone Ssz1 and the ribosomebinding Hsp40 cochaperone zuotin (Hsp70L1 and Mpp11 in mammals) (43). RAC cooperates with the ribosome-binding isoforms of Hsp70, Ssb1 and Ssb2 (50), and has been suggested to 
couple cotranslational folding with the mechanics of peptide elongation by the ribosome (51, 52). NAC, a dimeric complex of $\alpha-(31 \mathrm{kDa})$ and $\beta$ - $(22 \mathrm{kDa})$ subunits, associates with ribosomes via the $\beta$-subunit and binds short nascent chains. Recent findings in C. elegans show that protein aggregates sequester NAC, thereby reducing translational capacity and implicating NAC as a sensor of protein quality control status (53). Moreover, NAC is required for correct intracellular protein sorting (54-56). Specifically, NAC modulates the fidelity of the signal recognition particle (SRP) in targeting proteins to the endoplasmic reticulum (ER) (54-56). Moreover, mistargeting of mitochondrial proteins to the ER occurs when NAC function is impaired (56).

\section{Chaperone functions downstream of the ribosome}

For proteins that are unable to fold with the ribosome-associated chaperones ( $\geq 30 \%$ of the proteome), the next layer of folding assistance is provided by the Hsp70 system, which is abundant in bacteria and eukarya (Fig. 4). Multiple Hsp70 molecules may interact with a nascent chain, preventing unproductive inter-domain interactions and supporting cotranslational folding (23, 50, 57). In addition, Hsp70 functions as a hub, providing connections to the more specialized downstream chaperones, Hsp90 and chaperonins. Substrate-loaded Hsp70 is coupled to Hsp90 via the Hsp organizing protein (Hop), which physically connects Hsp70 to Hsp90 to facilitate client transfer $(58,59)$ (Fig. 4B). Hsp70 also interacts directly with the eukaryotic chaperonin TRiC/CCT (60) (Fig. 4B), and in E. coli maintains certain proteins in a foldingcompetent state before transfer to the chaperonin GroEL (23) (Fig. 4A). In addition to Hsp70, in eukarya the jellyfish-like, hexameric prefoldin (Pfd) can bind to nascent chains and mediates transfer to the chaperonin (Fig. 4B). In archaea, many species of which lack Hsp70, Pfd may fullfil a more general role as a nascent chain-binding chaperone (23). 


\section{Chaperone Paradigms}

Several mechanistic paradigms of chaperone function in protein folding have been defined (13).

In the following sections, we discuss major recent advances in understanding the mechanisms of the Hsp70, chaperonin and Hsp90 machineries.

The Hsp70 System

Hsp70 (DnaK in bacteria) is a paradigm allosteric chaperone that binds to 5-7 aa sequence elements that are enriched in hydrophobic residues and are typically exposed by non-native proteins $(61,62)$. ATP-dependent binding and release of such segments allows Hsp70s to participate in a wide range of cellular processes, including protein folding, refolding, disaggregation, and protein transfer to cellular compartments or the proteolytic machinery (61, $62)$.

Hsp70 has two domains: an N-terminal nucleotide-binding domain (NBD) of $\sim 40 \mathrm{kDa}$ and a C-terminal substrate-binding domain (SBD) of $\sim 30 \mathrm{kDa}$, connected by a hydrophobic linker (61) (Fig. 5A). The SBD consists of a $\beta$-sandwich domain, harboring the peptide binding site, and an $\alpha$-helical lid segment. Peptide substrate binds in an extended conformation in a groove in the $\beta$-sandwich domain (Fig. 5A).

The Hsp70 reaction cycle is intimately regulated by Hsp40 proteins and nucleotide exchange factors (NEFs) (Fig. 5B). Hsp40 cochaperones (DnaJ in bacteria, jointly known as Jdomain proteins) function in recognizing and transferring substrate proteins to Hsp70 in the ATP state, in which the hydrophobic inter-domain linker and the $\alpha$-helical lid of the SBD are associated with the NBD, and the SBD is in an open conformation (63-65). In this state Hsp70 has high on- and off-rates for substrate. Interaction of Hsp40 with Hsp70 strongly accelerates (by $>1000$-fold) the hydrolysis of the bound ATP, generating the ADP-state, in which SBD and 
NBD are loosely associated $(61,66)$ and the $\alpha$-helical lid is in the closed conformation, trapping the bound substrate (low on- and off-rates) $(61,67)$ (Fig. 5B). Subsequent NEF binding to the NBD facilitates ADP-ATP exchange, opening the SBD and allowing substrate release for folding or transfer to downstream chaperones or the degradation machinery. Rebinding prevents offpathway aggregation and can reverse misfolding by removing long-range interactions present in unbound folding intermediate (kinetic partitioning mechanism) (68-71). It has also been suggested that DnaK binding can bias the folding pathway such that the bound substrate first forms secondary structure, followed by the development of longer-range contacts upon release (71, 72). In metazoans the cycle is further regulated by the Hsp70 interacting protein Hip, which stabilizes Hsp70 in the ADP-state, delaying client protein release (73) (Fig. 5B).

Compared to bacteria, the number of Hsp40 proteins is markedly expanded in eukarya. Human cells express approximately 50 different J-domain proteins (74), amplifying the ability of Hsp70 to recognize a diverse range of clients. For example, Hsp70 cooperates with the Hsp40 auxilin in disassembling clathrin cages of endocytic vesicles (62) and with other specific Hsp40s in supporting the lifecycle of certain viruses (75). The NEFs of Hsp70 are less diverse (76). Bacteria contain only a single NEF, GrpE, while several structurally unrelated families of NEFs exist in eukarya, including the Bcl-2-associated anthanogene (BAG) domain proteins, as well as HspBP1 and Hsp1 10 (76). The Hsp110 proteins are Hsp70 homologs that function also as holding chaperones and cooperate with Hsp70 in protein disaggregation $(77,78)$. In eukarya the C-terminus of cytosolic Hsp70 is unstructured and mediates interactions between Hsp70 and Hsp90 (via Hop) or the ubiquitin-proteasome system (via CHIP) (61, 79) (Fig. 5B). Both Hop and CHIP contain tetratricopeptide repeat (TPR) domains that bind the C-terminus of Hsp70 and Hsp90 (13). 
Chaperonins - nano-cages for protein folding

The chaperonins (Hsp60s) are multi-subunit cylindrical complexes that function by enclosing a single molecule of non-native protein in their central cavity for folding to occur unimpaired by aggregation. They are essential in all three domains of life and are divided into two groups.

Group I chaperonins occur in the bacterial cytosol (GroEL), mitochondria (Hsp60), and chloroplasts (Cpn60) (80), group II chaperonins in archaea (thermosome) and the eukaryotic cytosol (TRiC, also known as CCT) (81). The two chaperonin classes share a common subunit organization and general architecture, both forming 1 MDa complexes consisting of two rings of 7-9 subunits of $\sim 60 \mathrm{kDa}$ that are stacked back-to-back. Group I and II chaperonins are unable to fold client proteins interchangeably, implying important differences in substrate protein recognition or folding mechanism.

The group I chaperonin GroEL of the bacterial cytosol is involved in the folding of $\sim 10 \%$ of the E.coli proteome including proteins that cannot use the upstream chaperones for folding $(80,82)$. GroEL cooperates with the lid-shaped GroES, a heptameric ring of $\sim 10 \mathrm{kDa}$ subunits that binds to the ends of the GroEL cylinder (Fig. 6A). The GroEL subunits contain an equatorial ATPase domain, an intermediate hinge-domain and an apical domain (82) (Fig. 6A). The apical domains form the entrance to the cavity and expose hydrophobic residues for the binding of a non-native substrate protein. The two rings of GroEL function sequentially as folding chambers, regulated allosterically by the GroEL ATPase $(83,84)$. Binding of ATP and GroES to the substrate-bound ring causes the displacement of the non-native substrate from its hydrophobic attachment sites into a cavity capped by GroES (Fig. 6B). This step is accompanied by extensive conformational changes of the GroEL subunits that enlarge the ring cavity and alter its physical properties from hydrophobic to hydrophilic $(80,82)$. Proteins up to $\sim 60 \mathrm{kDa}$ in size are now free to fold in the chaperonin nano-cage for the time it takes GroEL to hydrolyze its bound ATP to 
$\operatorname{ADP}\left(\sim 2 \mathrm{sec}\right.$ at $\left.37^{\circ} \mathrm{C}\right)(85)$. Binding of ATP to the opposite GroEL ring then induces an allosteric signal that causes ADP to dissociate and GroES to unbind. Folded protein is released, while incompletely folded/misfolded molecules may rebind, resulting in possible unfolding in preparation for another folding cycle $(86,87)$. Whether the GroEL rings function strictly sequentially in folding or sometimes in parallel, with both folding chambers being capped by GroES simultaneously, is a matter of ongoing research $(80,84,88)$.

The GroEL-dependent proteins tend to populate aggregation-prone, kinetically trapped folding intermediates when folding in free solution $(89,90)$. While aggregation prevention is an important function of the chaperonin (91), recent studies show that GroEL/GroES can markedly accelerate the folding of some of these proteins by smoothing their folding energy landscape (85, 92-96). The confining environment of the chaperonin cage allows complex topologies to form sequentially, thereby guiding the substrate along a folding pathway that avoids kinetic traps (95). Exactly how GroEL/GroES accelerates protein folding is not yet clear, but has been shown to depend on the volume of the cage, the negative charge of the cavity wall and the flexible Ctermini of the GroEL subunits that protrude into the cavity (80).

The eukaryotic chaperonin TRiC also supports the folding of $\sim 10 \%$ of the proteome, including the key cytoskeletal proteins actin and tubulin (81). Like GroEL, TRiC cycles between open and closed states, but substrate encapsulation is cofactor-independent and is mediated by helical protrusions from the tip of each subunit that function as an in-built lid (97) (Fig. 6C). The iris-like lid does not seal the cavity completely, but leaves a small pore that can accommodate extended polypeptide chains. This feature allows TRiC to encapsulate and fold large substrates in a domain-wise fashion and act cotranslationally (98) (Fig. 4B). Unlike the homo-oligomeric GroEL, each ring of TRiC consists of eight paralogous subunits, which differ in substrate 
recognition. Thus, TRiC may dictate the topology of the bound substrate (99) (Fig. 6D). The subunits also vary in their affinity for ATP, with low- and high-affinity subunits being spatially segregated within the ring (100). As a result, the ATP-driven conformational cycle may proceed sequentially (101). Moreover, the unique subunit arrangement creates a pronounced asymmetry in the distribution of charges in the folding chamber (102-104) (Fig. 6D), which may influence the folding trajectory of the encapsulated substrate. The TRiC-mediated folding of certain substrates, such as G-protein $\beta$ subunits, is enhanced by cooperation with the co-chaperone phosducin-like protein (105). Although the structure and conformational cycle of TRiC are now well understood, it remains unclear how exactly the special features of the eukaryotic chaperonin contribute to protein folding. Hsp90 - conformational surveillance of metastable clients In eukaryotes Hsp90 supports not only protein folding, but also the conformational maturation and maintenance of a range of medically relevant signaling proteins, including proto-oncogenic kinases, steroid hormone receptors and transcription factors (106-110). Owing to its involvement in multiple signaling pathways, Hsp90 also plays an important evolutionary role by buffering destabilizing mutations in its client proteins (111).

Hsp90 is a homodimer, with each subunit consisting of an N-terminal nucleotide binding domain (NTD) of $\sim 25 \mathrm{kDa}$, a middle domain (MD) of $\sim 40 \mathrm{kDa}$, and a C-terminal dimerization domain (CTD) of $\sim 12 \mathrm{kDa}(107,112,113)$ (Fig. 7A). A charged linker region connects the NTD with the MD. The Hsp90 dimer is dynamic and samples a range of conformations that are selectively stabilized by nucleotide, client protein and various cofactors $(114,115)$. In the absence of nucleotide, Hsp90 favors an open conformation (Fig. 7A and B). Upon ATP binding, a lid segment of the NTD closes over the bound nucleotide, which leads to NTD dimerization via strand exchange. A flexible loop from the MD contacts the NTD inducing a twisted closed 
conformation, with residues from the MD contributing to ATP hydrolysis (112) (Fig. 7A and B). After ATP hydrolysis and nucleotide release Hsp90 reverts to the open state (Fig. 7B). Although ATP contributes free energy and directionality to the Hsp90 conformational cycle, the rate limiting step is not ATP hydrolysis, but the large conformational transition from the open to closed state (116).

How Hsp90 recognizes substrates and how its ATPase cycle is coupled to client maturation is not yet well understood. Recent evidence suggests that Hsp90 has an extended substrate binding interface that crosses domain boundaries and exhibits specificity for proteins with exposed hydrophobic residues, independent of whether they are disordered, partly folded, or near native $(113,117,118)$. Interaction with Hsp90 can selectively stabilize metastable client proteins in their active conformation, as shown for glucocorticoid receptor and v-Src kinase (58, 119), explaining why Hsp90 is especially important in cancer cells where growth is driven by the constitutive activation of one or more oncoproteins.

Hsp90 cooperates with various cochaperones, which regulate its ATPase activity and recruit clients $(107,112)$ (Fig. 7B). These factors act sequentially along the Hsp90 cycle, and in some cases form mixed complexes with the chaperone (120). Hop and Cdc37 stabilize the open conformation of the Hsp90 dimer, inhibit ATP hydrolysis and facilitate client binding. Hop mediates client transfer from Hsp70 to Hsp90, whereas Cdc37 functions as an adapter for kinase clients. Aha1 binds asymmetrically to the MD and NTD of the Hsp90 dimer, facilitating transition to the closed conformation and thereby accelerating ATP hydrolysis $(121,122)$. p23 acts towards the end of the cycle, and facilitates client maturation by stabilizing the closed state of the Hsp90 N-domains and inhibiting ATP hydrolysis (107). In addition, Hsp90 cooperates with a range of other cofactors containing TPR-domains. Some of these factors also contain 
peptidyl-prolyl-isomerase (PPIase) domains and participate in client folding on Hsp90 (109). In contrast to eukaryotic Hsp90, the bacterial Hsp90, HtpG, is independent of Hsp90 cochaperones.

\section{The Proteostasis Network}

Proteostasis, the state in which the proteome of an organism is in functional balance, must be tightly controlled within individual cells, tissues and organs. To maintain proteome integrity, the different chaperone classes and their cofactors cooperate with the machineries of protein synthesis and protein degradation in a coordinated proteostasis network (PN), comprising $\sim 1400$ components in mammalian cells (123-125) (Fig. 8A). The PN must balance protein synthesis with turnover, clear surplus, misfolded and aggregated proteins, and respond to stress. Importantly, decline or disruption of the PN is strongly associated with aging and diseases of aberrant protein folding and aggregation $(4,126)$. The organization of the PN appears to be robust, displaying a high degree of redundancy. For example, only 55 of the 332 chaperones were found to be among the core fitness genes of human leukemia K562 cells $(123,127)$ (Fig. 9). In C. elegans, knockdown of only 20 of the 219 chaperones enhanced aggregation and toxicity of neurodegenerative disease proteins (123). The large size of the PN with in-built redundancy would facilitate adaptation to the needs of specific cell types and tissues, dependent on environmental and physical exposure, as well as requirements of the expressed proteome.

Stress conditions, including exposure to elevated temperature or oxidizing agents, upregulate multiple chaperones (stress proteins) by inducing transcriptional programs dedicated to specific cell compartments. These include the cytosolic stress response (128) and the unfolded protein response (UPR) pathways of the ER and mitochondria $(129,130)$. The stress response of individual cell types and tissues is further coordinated at the organismal level by cell nonautonomous signaling mechanisms $(4,131)$. 
Degradation of terminally misfolded proteins and control of protein stoichiometries is mainly performed by the UPS $(132,133)$, which comprises several hundred components $(133)$ and plays an important role in maintaining proteome balance (134) (Fig. 8A). Interestingly, certain misfolded proteins require chaperone-mediated transport from the cytosol into the nucleus for proteasomal degradation (135-137), defining the nucleus as a general protein quality control compartment. Clearance of aggregated proteins by the UPS requires disaggregation prior to degradation, which is performed by Hsp70 in cooperation with Hsp110 and a mixed-class Hsp40 complex $(77,78)$ (Fig. 8A). In bacteria and fungi, AAA+ ATPase complexes of the Hsp100 family cooperate with the Hsp70 system in disaggregation (138). Protein aggregates resisting disassembly may be cleared by selective autophagy and lysosomal degradation (133) (Fig. 8A). Both proteasomal and lysosomal clearance pathways are tightly coupled to the Hsp70 and Hsp90 chaperone systems via BAG-domain proteins and specific ubiquitin ligases, such as the cochaperone CHIP $(139,140)$.

\section{Toxicity of protein misfolding and aggregation}

Protein dysfunction resulting from misfolding due to mutation (single nucleotide polymorphisms, SNPs) renders proteins metastable and underlies many pathologies, including cystic fibrosis and a wide range of metabolic defects (141). While a fully functional PN may buffer the destabilizing effects of SNPs on protein conformation, metastable mutant proteins tend to aggregate when PN capacity is overwhelmed, as occurs progressively during aging $(142,143)$. Age-dependent decline of the PN facilitates the toxic aggregation of neurodegenerative disease proteins (both wild-type and mutant forms), such as amyloid beta peptide (A $\beta$ ) and Tau in Alzheimer's disease, $\alpha$-synuclein in Parkinson's disease and huntingtin in Huntington's disease. The hallmark of these pathologies is the intra- or extracellular deposition of aggregates containing amyloid-like $\beta$-sheet fibrils (Fig. 8A). Chaperones have been shown to interfere at 
various steps of the aggregation cascade (144), including primary nucleation, fibril elongation and fragmentation, and secondary nucleation by the fibril surface $(145,146)$. Toxic aggregates are also produced when faulty translation products accumulate (147-149), for example when polypeptide chains that stall during translation on the ribosome are not efficiently cleared by ribosome quality control (RQC) machinery and the UPS (149-151).

The toxicity arising from aggregation underlies common structural properties of the aggregates (gain of toxic function) and may be entirely unrelated to the normal function of the affected protein. In parallel with assembling to insoluble end-stage fibrils, the disease proteins often accumulate toxic oligomers that expose 'sticky' surfaces (hydrophobic amino acid residues and unpaired $\beta$-strands) (152). These features confer to the oligomers the ability to engage in aberrant interactions with multiple key cellular proteins $(153,154)$. The proteins targeted by toxic aggregates are often themselves metastable $(155,156)$; they are typically enriched in intrinsically unstructured regions and sequences of low amino acid complexity, characteristic features of many RNA binding proteins (154-157). Toxic protein aggregation interferes with nucleo-cytoplasmic transport of RNA and protein, and generally impairs RNA homeostasis (156, 158). Moreover, the aggregates impair protein degradation by the proteasome and autophagy systems (124) and sequester critical components of the chaperone network, such as certain Hsp40 cochaperones $(137,149,151)$. Protein aggregation therefore directly interferes with PN function, and is both a symptom and cause of PN decline - setting in motion a vicious cycle that ultimately triggers proteostasis collapse (Fig. 8B).

Aggregate management is critical in determining cell viability and the lifespan of model organisms. Proteins have been optimized during evolution to maintain solubility at their physiological concentrations (159). Thus, even a modest increase in abundance, as might be 
caused by age-dependent proteome imbalance or due to aneuploidy $(160,161)$, will result in loss of solubility. Interestingly, recent evidence indicates that cells contain machineries that actively sequester surplus and misfolded proteins into transient or stable deposits when their timely degradation fails (161-168), presumably an attempt to reduce toxic effects exerted by soluble oligomers (169).

\section{The $P N$ as a drug target}

Mechanisms that promote proteostasis have the potential to maintain mutant proteins in a functional, non-aggregated state, slow aging and decrease the incidence of age-related diseases in model organisms. Thus, boosting PN capacity may be beneficial in both loss-of-function and gain-of-toxic function diseases of protein misfolding (170). Conversely, attenuating the PN by inhibiting specific chaperone or degradation pathways is effective in cancer treatment, based on the fact that oncogenic proteins are often metastable and thus highly chaperone dependent $(110$, 124, 171, 172). For example, the inhibition of Hsp90 with molecules that block ATP binding prevents the activation of client proteins that promote tumor growth and directs them towards degradation $(110,171)$. Similarly, pharmacologic inhibition of Hsp70 or its co-factor interactions may interfere with folding and promote protein degradation, defining a promising strategy in the treatment of cancer and certain neurodegenerative diseases (172).

In principle, PN capacity can be enhanced in several ways (Fig 8A): i) By downregulating translation to reduce the load of misfolded proteins on the chaperone system, ii) by upregulating chaperone levels to improve folding and conformational repair, and iii) by enhancing the clearance of misfolded proteins and aggregates or by promoting the formation of inclusions that are non-toxic. For example, the antihypertensive drug Guanabenz has the additional effect of attenuating translation by maintaining translation initiation factor 2 (eIF $2 \alpha$ ) in its inactive, phosphorylated state $(173,174)$, which mimics the transient inhibition of 
translation upon unfolded protein stress. On the other hand, certain neuronal cells are highly sensitive to translational attenuation (175) and the pharmacologic reversal of eIF2 $\alpha$ phosphorylation has been shown to improve cognitive memory in mice (176-178). Numerous small molecule compounds have been described that activate cellular stress response pathways and reduce the aggregation of disease proteins or enhance their clearance by increasing chaperone levels $(13,179-181)$. A key role in aggregation prevention and aggregate dissociation is played by the Hsp70 machinery, as shown by overexpressing single or multiple components of the system in cellular and animal disease models $(124,154,182,183)$. Finally, activating the UPS or inducing autophagy with small molecule compounds can enhance degradation and reduce aggregate load $(184,185)$. Much excitement is caused by the recent development and approval of the first proteostasis modulator compounds for specific proteins. These include

therapeutics for cystic fibrosis that specifically target the folding and trafficking defect of mutant CFTR (186) and drugs for the treatment of transthyretin amyloidoses, fatal aggregation diseases characterized by progressive neuropathy and cardiomyophathy (187).

\section{Conclusions}

Since the emergence of the molecular chaperone concept more than two decades ago, dramatic progress has been made towards understanding protein folding in vivo and how cells maintain proteome balance. The resulting field is exerting a major influence on several other areas, from basic biology to medicine. A number of intriguing questions remain, however, regarding both the mechanism of individual chaperone machines, and how their functions are coordinated within the PN. 
From a mechanistic perspective, it remains unclear how exactly the major ATPdependent chaperone systems (Hsp70, Hsp90, chaperonins) support protein folding. Resolving this question will require detailed molecular descriptions of the folding pathways of respective client proteins. For example, it will be fascinating to discover why the eukaryotic chaperonin TRiC can fold proteins, like actin and tubulins, which the bacterial GroEL/GroES is unable to handle. The contribution of the ribosome to protein folding is another intriguing subject of current research. Going forward, it will be important to learn how the environment of the ribosome exit channel affects the energy landscape of cotranslational folding, and whether this effect is a generic feature of folding in vivo. The confinement of small polypeptides within the negatively charged ribosome exit channel is reminiscent of the effect of the chaperonin folding cage, and the two systems may influence folding in similar ways, albeit for proteins of vastly different sizes.

The interactions between different chaperone systems and other components of the PN remain largely unexplored. In particular, little is known about how chaperone systems respond to increased load due to proteotoxic stress, and how cells achieve an optimum balance between different chaperone activities (e.g. folding versus holding and aggregation prevention, or transfer to the degradation machinery). The crosstalk between compartment specific stress response pathways is another area of interest. A holistic understanding of the PN will be vital to future attempts to manipulate its activities pharmacologically, and should offer new opportunities to treat the wide range of diseases associated with declining proteostasis. 


\section{$\underline{\text { References }}$}

1. W. E. Balch, R. I. Morimoto, A. Dillin, J. W. Kelly, Adapting proteostasis for disease intervention. Science 319, 916-919 (2008).

2. A. Gershenson, L. M. Gierasch, A. Pastore, S. E. Radford, Energy landscapes of functional proteins are inherently risky. Nat. Chem. Biol. 10, 884-891 (2014).

3. A. K. Dunker, I. Silman, V. N. Uversky, J. L. Sussman, Function and structure of inherently disordered proteins. Curr. Opin. Struct. Biol. 18, 756-764 (2008).

4. J. Labbadia, R. I. Morimoto, The biology of proteostasis in aging and disease. Annu. Rev. Biochem. 84, 435-464 (2015).

5. I. Braakman, D. N. Hebert, Protein folding in the endoplasmic reticulum. Cold Spring Harb. Perspect. Biol. 5, a013201 (2013).

6. C. B. Anfinsen, Principles that govern the folding of protein chains. Science 181, 223-230 (1973).

7. F. U. Hartl, Molecular chaperones in cellular protein folding. Nature 381, 571-579 (1996).

8. D. J. Brockwell, S. E. Radford, Intermediates : ubiquitous species on folding energy landscapes? Curr. Opin. Struct. Biol. 17, 30-37 (2007).

9. R. J. Ellis, A. P. Minton, Protein aggregation in crowded environments. Biol. Chem. 387, 485-497 (2006).

10. A. R. Dinner, A. Sali, L. J. Smith, C. M. Dobson, M. Karplus, Understanding protein folding via free-energy surfaces from theory and experiment. Trends Biochem. Sci. 25, 331-339 (2000).

11. P. A. Schmidpeter, F. X. Schmid, Prolyl isomerization and its catalysis in protein folding and protein function. J. Mol. Biol. 427, 1609-1631 (2015).

12. F. Chiti, C. M. Dobson, Protein misfolding, functional amyloid, and human disease. Annu. Rev. Biochem. 75, 333-366 (2006).

13. Y. E. Kim, M. S. Hipp, A. Bracher, M. Hayer-Hartl, F. U. Hartl, Molecular chaperone functions in protein folding and proteostasis. Annu. Rev. Biochem. 82, 323-355 (2013).

14. D. N. Wilson, R. Beckmann, The ribosomal tunnel as a functional environment for nascent polypeptide folding and translational stalling. Curr. Opin. Struct. Biol. 21, 274282 (2011).

15. A. Kosolapov, C. Deutsch, Tertiary interactions within the ribosomal exit tunnel. Nat. Struct. Mol. Biol. 16, 405-411 (2009).

16. E. P. O’Brien, J. Christodoulou, M. Vendruscolo, C. M. Dobson, New scenarios of protein folding can occur on the ribosome. J. Amer. Chem. Soc. 133, 513-526 (2011).

17. O. B. Nilsson et al., Cotranslational protein folding inside the ribosome exit tunnel. Cell Rep. 12, 1533-1540 (2015). 
18. W. Holtkamp et al., Cotranslational protein folding on the ribosome monitored in real time. Science 350, 1104-1107 (2015).

19. C. M. Kaiser, D. H. Goldman, J. D. Chodera, I. Tinoco, Jr., C. Bustamante, The ribosome modulates nascent protein folding. Science 334, 1723-1727 (2011).

20. L. D. Cabrita et al., A structural ensemble of a ribosome-nascent chain complex during cotranslational protein folding. Nat. Struct. Mol. Biol., doi: 10.1038/nsmb.3182 (2016).

21. C. Eichmann, S. Preissler, R. Riek, E. Deuerling, Cotranslational structure acquisition of nascent polypeptides monitored by NMR spectroscopy. Proc. Natl. Acad. Sci. U.S.A. 107, 9111-9116 (2010).

22. O. B. Nilsson, A. Muller-Lucks, G. Kramer, B. Bukau, G. von Heijne, Trigger factor reduces the force exerted on the nascent chain by a cotranslationally folding protein. $J$. Mol. Biol., doi: 10.1016/j.jmb.2016.02.014 (2016).

23. F. U. Hartl, M. Hayer-Hartl, Molecular chaperones in the cytosol: from nascent chain to folded protein. Science 295, 1852-1858 (2002).

24. Z. N. Scholl, W. Yang, P. E. Marszalek, Chaperones rescue luciferase folding by separating its domains. J. Biol. Chem. 289, 28607-28618 (2014).

25. A. Borgia et al., Transient misfolding dominates multidomain protein folding. Nature Commun. 6, 8861 (2015).

26. Y.-W. Shieh et al., Operon structure and cotranslational subunit association direct protein assembly in bacteria. Science 350, 678-680 (2015).

27. F. Brandt et al., The native 3D organization of bacterial polysomes. Cell 136, 261-271 (2009).

28. F. Brandt, L.-A. Carlson, F. U. Hartl, W. Baumeister, K. Grunewald, The threedimensional organization of polyribosomes in intact human cells. Mol. Cell 39, 560-569 (2010).

29. G. W. Li, E. Oh, J. S. Weissman, The anti-Shine-Dalgarno sequence drives translational pausing and codon choice in bacteria. Nature 484, 538-541 (2012).

30. S. Pechmann, J. Frydman, Evolutionary conservation of codon optimality reveals hidden signatures of cotranslational folding. Nat. Struct. Mol. Biol. 20, 237-243 (2012).

31. P. S. Spencer, E. Siller, J. F. Anderson, J. M. Barral, Silent substitutions predictably alter translation elongation rates and protein folding efficiencies. J. Mol. Biol. 422, 328-335 (2012).

32. E. P. O'Brien, M. Vendruscolo, C. M. Dobson, Kinetic modelling indicates that fasttranslating codons can coordinate cotranslational protein folding by avoiding misfolded intermediates. Nature Commun. 5, 1-11 (2014).

33. S. J. Kim et al., Translational tuning optimizes nascent protein folding in cells. Science 348, 444-448 (2015). 
34. F. Mohammad, C. J. Woolstenhulme, R. Green, A. R. Buskirk, Clarifying the translational pausing landscape in bacteria by ribosome profiling. Cell Rep. 14, 686-694 (2016).

35. M. Zhou et al., Non-optimal codon usage affects expression, structure and function of clock protein FRQ. Nature 495, 111-115 (2013).

36. C. Kimchi-Sarfaty et al., A "silent" polymorphism in the MDR1 gene changes substrate specificity. Science 315, 525-528 (2007).

37. R. M. Vabulas, F. U. Hartl, Protein synthesis upon acute nutrient restriction relies on proteasome function. Science 310, 1960-1963 (2005).

38. S. Duttler, S. Pechmann, J. Frydman, Principles of cotranslational ubiquitination and quality control at the ribosome. Mol. Cell 50, 379-393 (2013).

39. T. M. Treweek, S. Meehan, H. Ecroyd, J. A. Carver, Small heat-shock proteins: important players in regulating cellular proteostasis. Cell. Mol. Life Sci. 72, 429-451 (2015).

40. A. Chari, U. Fischer, Cellular strategies for the assembly of molecular machines. Trends Biochem. Sci. 35, 676-683 (2010).

41. T. Hauser, L. Popilka, F. U. Hartl, M. Hayer-Hartl, Role of auxiliary proteins in Rubisco biogenesis and function. Nature Plants 1, 1-11 (2015).

42. E. Oh et al., Selective ribosome profiling reveals the cotranslational chaperone action of trigger factor in vivo. Cell 147, 1295-1308 (2011).

43. S. Preissler, E. Deuerling, Ribosome-associated chaperones as key players in proteostasis. Trends Biochem. Sci. 37, 274-283 (2012).

44. C. M. Kaiser et al., Real-time observation of trigger factor function on translating ribosomes. Nature 444, 455-460 (2006).

45. A. Hoffmann et al., Concerted action of the ribosome and the associated chaperone trigger factor confines nascent polypeptide folding. Mol. Cell 48, 63-74 (2012).

46. A. Mashaghi et al., Reshaping of the conformational search of a protein by the chaperone trigger factor. Nature 500, 98-101 (2013).

47. T. Saio, X. Guan, P. Rossi, A. Economou, C. G. Kalodimos, Structural basis for protein antiaggregation activity of the trigger factor chaperone. Science 344, 1250494 (2014).

48. V. R. Agashe et al., Function of trigger factor and DnaK in multidomain protein folding: increase in yield at the expense of folding speed. Cell 117, 199-209 (2004).

49. F. Stull, P. Koldewey, J. R. Humes, S. E. Radford, J. C. Bardwell, Substrate protein folds while it is bound to the ATP-independent chaperone Spy. Nat. Struct. Mol. Biol. 23, 53-58 (2016).

50. F. Willmund et al., The cotranslational function of ribosome-associated Hsp70 in eukaryotic protein homeostasis. Cell 152, 196-209 (2013).

51. M. Chiabudini, C. Conz, F. Reckmann, S. Rospert, Ribosome-associated complex and Ssb are required for translational repression induced by polylysine segments within nascent 
chains. Mol. Cell. Biol. 32, 4769-4779 (2012).

52. Y. Zhang et al., Structural basis for interaction of a cotranslational chaperone with the eukaryotic ribosome. Nat. Struct. Mol. Biol. 21, 1042-1046 (2014).

53. J. Kirstein-Miles, A. Scior, E. Deuerling, R. I. Morimoto, The nascent polypeptideassociated complex is a key regulator of proteostasis. EMBO J. 32, 1451-1468 (2013).

54. M. del Alamo et al., Defining the specificity of cotranslationally acting chaperones by systematic analysis of mRNAs associated with ribosome-nascent chain complexes. PLoS Biol. 9, e1001100 (2011).

55. Y. Zhang et al., NAC functions as a modulator of SRP during the early steps of protein targeting to the endoplasmic reticulum. Mol. Biol. Cell 23, 3027-3040 (2012).

56. M. Gamerdinger, M. A. Hanebuth, T. Frickey, E. Deuerling, The principle of antagonism ensures protein targeting specificity at the endoplasmic reticulum. Science 348, 201-207 (2015).

57. G. Calloni et al., DnaK functions as a central hub in the E. coli chaperone network. Cell Rep. 1, 251-264 (2012).

58. E. Kirschke, D. Goswami, D. Southworth, P. R. Griffin, D. A. Agard, Glucocorticoid receptor function regulated by coordinated action of the Hsp90 and Hsp70 chaperone cycles. Cell 157, 1685-1697 (2014).

59. N. Morgner et al., Hsp70 forms antiparallel dimers stabilized by post-translational modifications to position clients for transfer to Hsp90. Cell Rep. 11, 759-769 (2015).

60. J. Cuellar et al., The structure of CCT-Hsc70 NBD suggests a mechanism for Hsp70 delivery of substrates to the chaperonin. Nat. Struct. Mol. Biol. 15, 858-864 (2008).

61. M. P. Mayer, Hsp70 chaperone dynamics and molecular mechanism. Trends Biochem. Sci. 38, 507-514 (2013).

62. E. M. Clerico, J. M. Tilitsky, W. Meng, L. M. Gierasch, How Hsp70 molecular machines interact with their substrates to mediate diverse physiological functions. J. Mol. Biol. 427, 1575-1588 (2015).

63. R. Kityk, J. Kopp, I. Sinning, M. P. Mayer, Structure and dynamics of the ATP-bound open conformation of Hsp70 chaperones. Mol. Cell 48, 863-874 (2012).

64. R. Qi et al., Allosteric opening of the polypeptide-binding site when an Hsp70 binds ATP. Nat. Struct. Mol. Biol. 20, 900-907 (2013).

65. A. Zhuravleva, L. M. Gierasch, Substrate-binding domain conformational dynamics mediate Hsp70 allostery. Proc. Natl. Acad. Sci. U.S.A. 112, E2865-2873 (2015).

66. M. Sikor, K. Mapa, L. V. von Voithenberg, D. Mokranjac, D. C. Lamb, Real-time observation of the conformational dynamics of mitochondrial Hsp70 by spFRET. EMBO J. 32, 1639-1649 (2013).

67. P. De Los Rios, A. Barducci, Hsp70 chaperones are non-equilibrium machines that 
achieve ultra-affinity by energy consumption. eLife 3, e02218 (2014).

68. S. K. Sharma, P. De Los Rios, P. Christen, A. Lustig, P. Goloubinoff, The kinetic parameters and energy cost of the Hsp70 chaperone as a polypeptide unfoldase. Nat. Chem. Biol. 6, 914-920 (2010).

69. J. M. Nunes, M. Mayer-Hartl, F. U. Hartl, D. J. Muller, Action of the Hsp70 chaperone system observed with single proteins. Nat. Commun. 6, 6307 (2015).

70. A. Sekhar, R. Rosenzweig, G. Bouvignies, L. E. Kay, Mapping the conformation of a client protein through the Hsp70 functional cycle. Proc. Natl. Acad. Sci. U.S.A. 112, 10395-10400 (2015).

71. A. Sekhar, R. Rosenzweig, G. Bouvignies, L. E. Kay, Hsp70 biases the folding pathways of client proteins. Proc. Natl. Acad. Sci. U.S.A. DOI: 10.1073/pnas.1601846113 (2016).

72. J. H. Lee et al., Heterogeneous binding of the SH3 client protein to the DnaK molecular chaperone. Proc. Natl. Acad. Sci. U.S.A. 112, E4206-4215 (2015).

73. Z. Li, F. U. Hartl, A. Bracher, Structure and function of Hip, an attenuator of the Hsp70 chaperone cycle. Nat. Struct. Mol. Biol. 20, 929-935 (2013).

74. H. H. Kampinga, E. A. Craig, The Hsp70 chaperone machinery: J proteins as drivers of functional specificity. Nat. Rev. Mol. Cell Biol. 11, 579-592 (2010).

75. S. Taguwa et al., Defining Hsp70 subnetworks in dengue virus replication reveals key vulnerability in flavivirus infection. Cell 163, 1108-1123 (2015).

76. A. Bracher, J. Verghese, The nucleotide exchange factors of Hsp70 molecular chaperones. Front. Mol. Biosci. 2, 10 (2015).

77. H. Rampelt et al., Metazoan Hsp70 machines use Hsp110 to power protein disaggregation. EMBO J. 31, 4221-4235 (2012).

78. N. B. Nillegoda et al., Crucial Hsp70 co-chaperone complex unlocks metazoan protein disaggregation. Nature 524, 247-251 (2015).

79. H. Zhang et al., A bipartite interaction between Hsp70 and CHIP regulates ubiquitination of chaperoned client proteins. Structure 23, 472-482 (2015).

80. M. Hayer-Hartl, A. Bracher, F. U. Hartl, The GroEL-GroES chaperonin machine: A nanocage for protein folding. Trends Biochem. Sci. 41, 62-76 (2016).

81. T. Lopez, K. Dalton, J. Frydman, The mechanism and function of Group II chaperonins. J. Mol. Biol. 427, 2919-2930 (2015).

82. H. R. Saibil, W. A. Fenton, D. K. Clare, A. L. Horwich, Structure and allostery of the chaperonin GroEL. J. Mol. Biol. 425, 1476-1487 (2013).

83. D. K. Clare et al., ATP-triggered conformational changes delineate substrate-binding and -folding mechanics of the GroEL chaperonin. Cell 149, 113-123 (2012).

84. R. Gruber, A. Horovitz, Allosteric mechanisms in chaperonin machines. Chem. Rev., doi: 10.1021/acs.chemrev.5b00556 (2016). 
85. A. J. Gupta, S. Haldar, G. Miličić, F. U. Hartl, M. Hayer-Hartl, Active cage mechanism of chaperonin-assisted protein folding demonstrated at single-molecule level. J. Mol. Biol. 426, 2739-2754 (2014).

86. Z. Lin, D. Madan, H. S. Rye, GroEL stimulates protein folding through forced unfolding. Nat. Struct. Mol. Biol. 15, 303-311 (2008).

87. S. Sharma et al., Monitoring protein conformation along the pathway of chaperoninassisted folding. Cell 133, 142-153 (2008).

88. H. Taguchi, Reaction cycle of chaperonin GroEL via symmetric "football" intermediate. $J$. Mol. Biol. 427, 2912-2918 (2015).

89. M. J. Kerner et al., Proteome-wide analysis of chaperonin-dependent protein folding in Escherichia coli. Cell 122, 209-220 (2005).

90. K. Fujiwara, Y. Ishihama, K. Nakahigashi, T. Soga, H. Taguchi, A systematic survey of in vivo obligate chaperonin-dependent substrates. EMBO J. 29, 1552-1564 (2010).

91. A. L. Horwich, A. C. Apetri, W. a. Fenton, The GroEL/GroES cis cavity as a passive antiaggregation device. FEBS Lett. 583, 2654-2662 (2009).

92. Y.-C. Tang et al., Structural features of the GroEL-GroES nano-cage required for rapid folding of encapsulated protein. Cell 125, 903-914 (2006).

93. K. Chakraborty et al., Chaperonin-catalyzed rescue of kinetically trapped states in protein folding. Cell 142, 112-122 (2010).

94. A. L. Mallam, S. E. Jackson, Knot formation in newly translated proteins is spontaneous and accelerated by chaperonins. Nat. Chem. Biol. 8, 147-153 (2012).

95. F. Georgescauld et al., GroEL/ES chaperonin modulates the mechanism and accelerates the rate of TIM-barrel domain folding. Cell 157, 922-934 (2014).

96. J. Weaver, H. S. Rye, The C-terminal tails of the bacterial chaperonin GroEL stimulate protein folding by directly altering the conformation of a substrate protein. J. Biol. Chem. 289, 23219-23232 (2014).

97. N. R. Douglas et al., Dual action of ATP hydrolysis couples lid closure to substrate release into the group II chaperonin chamber. Cell 144, 240-252 (2011).

98. F. Rüßmann et al., Folding of large multidomain proteins by partial encapsulation in the chaperonin TRiC/CCT. Proc. Natl. Acad. Sci. U.S.A. 109, 21208-21215 (2012).

99. L. A. Joachimiak, T. Walzthoeni, C. W. Liu, R. Aebersold, J. Frydman, The structural basis of substrate recognition by the eukaryotic chaperonin TRiC/CCT. Cell 159, 10421055 (2014).

100. S. Reissmann et al., A gradient of ATP affinities generates an asymmetric power stroke driving the chaperonin TRiC/CCT folding cycle. Cell 2, 866-877 (2012).

101. D. Rivenzon-Segal, S. G. Wolf, L. Shimon, K. R. Willison, A. Horovitz, Sequential ATPinduced allosteric transitions of the cytoplasmic chaperonin containing TCP-1 revealed by 
EM analysis. Nat. Struct. Mol. Biol. 12, 233-237 (2005).

102. N. Kalisman, C. M. Adams, M. Levitt, Subunit order of eukaryotic TRiC/CCT chaperonin by cross-linking, mass spectrometry, and combinatorial homology modeling. Proc. Natl. Acad. Sci. U.S.A. 109, 2884-2889 (2012).

103. A. Leitner et al., The molecular architecture of the eukaryotic chaperonin TRiC/CCT. Structure 20, 814-825 (2012).

104. N. Kalisman, G. F. Schroder, M. Levitt, The crystal structures of the eukaryotic chaperonin CCT reveal its functional partitioning. Structure 21, 540-549 (2013).

105. R. L. Plimpton et al., Structures of the G $\beta-C C T$ and PhLP1-G $\beta-C C T$ complexes reveal a mechanism for G-protein $\beta$-subunit folding and G $\beta \gamma$ dimer assembly. Proc. Natl. Acad. Sci. U.S.A. 112, 2413-2418 (2015).

106. M. Taipale, D. F. Jarosz, S. Lindquist, Hsp90 at the hub of protein homeostasis: emerging mechanistic insights. Nature reviews. Mol. Cell Bio. 11, 515-528 (2010).

107. J. Li, J. Soroka, J. Buchner, The Hsp90 chaperone machinery: conformational dynamics and regulation by co-chaperones. Biochim. Biophys. Acta 1823, 624-635 (2012).

108. K. Sharma et al., Quantitative proteomics reveals that Hsp90 inhibition preferentially targets kinases and the DNA damage response. Mol. Cell. Proteomics 11, M111.01465 (2012).

109. M. Taipale et al., Quantitative analysis of Hsp90-client interactions reveals principles of substrate recognition. Cell 150, 987-1001 (2012).

110. W. B. Pratt, J. E. Gestwicki, Y. Osawa, A. P. Lieberman, Targeting Hsp90/Hsp70-based protein quality control for treatment of adult onset neurodegenerative diseases. Annu. Rev. Pharmacol. Toxicol. 55, 353-371 (2015).

111. S. Lindquist, Protein folding sculpting evolutionary change. Cold Spring Harb. Symp. Quant. Biol. 74, 103-108 (2009).

112. A. Röhl, J. Rohrberg, J. Buchner, The chaperone Hsp90: changing partners for demanding clients. Trends Biochem. Sci. 38, 253-262 (2013).

113. G. E. Karagoz, S. G. D. Rudiger, Hsp90 interaction with clients. Trends Biochem. Sci. 40, 117-125 (2015).

114. M. Mickler, M. Hessling, C. Ratzke, J. Buchner, T. Hugel, The large conformational changes of Hsp90 are only weakly coupled to ATP hydrolysis. Nat. Struct. Mol. Biol. 16, 281-286 (2009).

115. T. O. Street, L. A. Lavery, D. A. Agard, Substrate binding drives large-scale conformational changes in the Hsp90 molecular chaperone. Mol. Cell 42, 96-105 (2011).

116. M. Hessling, K. Richter, J. Buchner, Dissection of the ATP-induced conformational cycle of the molecular chaperone Hsp90. Nat. Struct. Mol. Biol. 16, 287-293 (2009).

117. G. E. Karagoz et al., Hsp90-tau complex reveals molecular basis for specificity in 
chaperone action. Cell 156, 963-974 (2014).

118. T. O. Street et al., Elucidating the mechanism of substrate recognition by the bacterial Hsp90 molecular chaperone. J. Mol. Biol. 426, 2393-2404 (2014).

119. E. E. Boczek et al., Conformational processing of oncogenic v-Src kinase by the molecular chaperone Hsp90. Proc. Natl. Acad. Sci. U.S.A. 112, E3189-E3198 (2015).

120. J. Li, K. Richter, J. Buchner, Mixed Hsp90-cochaperone complexes are important for the progression of the reaction cycle. Nat. Struct. Mol. Biol. 18, 61-66 (2011).

121. A. V. Koulov et al., Biological and structural basis for Aha1 regulation of Hsp90 ATPase activity in maintaining proteostasis in the human disease cystic fibrosis. Mol. Biol. Cell 21, 871-884 (2010).

122. M. Retzlaff et al., Asymmetric activation of the Hsp90 dimer by its cochaperone Aha1. Mol. Cell 37, 344-354 (2010).

123. M. Brehme et al., A chaperome subnetwork safeguards proteostasis in aging and neurodegenerative disease. Cell Rep. 9, 1135-1150 (2014).

124. M. S. Hipp, S.-H. Park, F. U. Hartl, Proteostasis impairment in protein-misfolding and aggregation diseases. Trends Cell Biol. 24, 506-514 (2014).

125. M. Taipale et al., A quantitative chaperone interaction network reveals the architecture of cellular protein homeostasis pathways. Cell 158, 434-448 (2014).

126. R. C. Taylor, A. Dillin, Aging as an event of proteostasis collapse. Cold Spring Harb. Perspect. Biol. 3, a004440 (2011).

127. T. Wang et al., Identification and characterization of essential genes in the human genome. Science 350, 1096-1101 (2015).

128. J. Anckar, L. Sistonen, Regulation of HSF1 function in the heat stress response: implications in aging and disease. Annu. Rev. Biochem. 80, 1089-1115 (2011).

129. P. Walter, D. Ron, The unfolded protein response: from stress pathway to homeostatic regulation. Science 334, 1081-1086 (2011).

130. A. M. Schulz, C. M. Haynes, UPR(mt)-mediated cytoprotection and organismal aging. Biochim. Biophys. Acta 1847, 1448-1456 (2015).

131. R. Schinzel, A. Dillin, Endocrine aspects of organelle stress-cell non-autonomous signaling of mitochondria and the ER. Curr. Opin. Cell Biol. 33, 102-110 (2015).

132. A. Shemorry, C. S. Hwang, A. Varshavsky, Control of protein quality and stoichiometries by N-terminal acetylation and the N-end rule pathway. Mol. Cell 50, 540-551 (2013).

133. A. Ciechanover, Y. T. Kwon, Degradation of misfolded proteins in neurodegenerative diseases: therapeutic targets and strategies. Exp. Mol. Med. 47, e147 (2015).

134. D. Vilchez et al., Increased proteasome activity in human embryonic stem cells is regulated by PSMD11. Nature 489, 304-308 (2012). 
135. J. W. Heck, S. K. Cheung, R. Y. Hampton, Cytoplasmic protein quality control degradation mediated by parallel actions of the E3 ubiquitin ligases Ubr1 and San1. Proc. Natl. Acad. Sci. U.S.A. 107, 1106-1111 (2010).

136. R. Prasad, S. Kawaguchi, D. T. Ng, A nucleus-based quality control mechanism for cytosolic proteins. Mol. Biol. Cell 21, $2117-2127$ (2010).

137. S.-H. Park et al., PolyQ proteins interfere with nuclear degradation of cytosolic proteins by sequestering the Sis $1 p$ chaperone. Cell 154, 134-145 (2013).

138. A. Mogk, E. Kummer, B. Bukau, Cooperation of Hsp70 and Hsp100 chaperone machines in protein disaggregation. Front. Mol. Biosci. 2, 22 (2015).

139. M. Gamerdinger et al., Protein quality control during aging involves recruitment of the macroautophagy pathway by BAG3. EMBO J. 28, 889-901 (2009).

140. N. Kettern, M. Dreiseidler, R. Tawo, J. Höhfeld, Chaperone-assisted degradation: multiple paths to destruction. Biol. Chem. 391, $481-489$ (2010).

141. N. Sahni et al., Widespread macromolecular interaction perturbations in human genetic disorders. Cell 161, 647-660 (2015).

142. A. Ben-Zvi, E. a. Miller, R. I. Morimoto, Collapse of proteostasis represents an early molecular event in Caenorhabditis elegans aging. Proc. Natl. Acad. Sci. U.S.A. 106, 14914-14919 (2009).

143. R. Gupta et al., Firefly luciferase mutants as sensors of proteome stress. Nat. Methods $\mathbf{8}$, 879-884 (2011).

144. T. P. Knowles et al., An analytical solution to the kinetics of breakable filament assembly. Science 326, 1533-1537 (2009).

145. G. Schaffar et al., Cellular toxicity of polyglutamine expansion proteins: Mechanism of transcription factor deactivation. Mol. Cell 15, 95-105 (2004).

146. S. I. Cohen et al., A molecular chaperone breaks the catalytic cycle that generates toxic A $\beta$ oligomers. Nat. Struct. Mol. Biol. 22, 207-213 (2015).

147. D. D. Nedialkova, S. A. Leidel, Optimization of codon translation rates via tRNA modifications maintains proteome integrity. Cell 161, 1606-1618 (2015).

148. S. Preissler et al., Not4-dependent translational repression is important for cellular protein homeostasis in yeast. EMBO J. 34, 1905-1924 (2015).

149. Y.-J. Choe, Park, S.-H., Hassemer, T., Korner, R., Vincenz-Donnelly, L., Hayer-Hartl, M. Hartl, F.U. , Failure of RQC machinery causes protein aggregation and proteotoxic stress. Nature 531, 191-195 (2016).

150. O. Brandman, R. S. Hegde, Ribosome-associated protein quality control. Nat. Struct. Mol. Biol. 23, 7-15 (2016).

151. R. Yonashiro et al., The Rqc2/Tae2 subunit of the ribosome-associated quality control (RQC) complex marks ribosome-stalled nascent polypeptide chains for aggregation. eLife 
5, e11794 (2016).

152. L. Breydo, V. N. Uversky, Structural, morphological, and functional diversity of amyloid oligomers. FEBS Lett. 589, 2640-2648 (2015).

153. K. L. Sugars, D. C. Rubinsztein, Transcriptional abnormalities in Huntington disease. Trends Genet. 19, 233-238 (2003).

154. H. Olzscha et al., Amyloid-like aggregates sequester numerous metastable proteins with essential cellular functions. Cell 144, 67-78 (2011).

155. A. Patel et al., A liquid-to-solid phase transition of the ALS protein FUS accelerated by disease mutation. Cell 162, 1066-1077 (2015).

156. A. C. Woerner et al., Cytoplasmic protein aggregates interfere with nucleocytoplasmic transport of protein and RNA. Science 351, 173-176 (2016).

157. S. Calabretta, S. Richard, Emerging roles of disordered sequences in RNA-binding proteins. Trends Biochem. Sci. 40, 662-672 (2015).

158. B. D. Freibaum et al., GGGGCC repeat expansion in C9orf72 compromises nucleocytoplasmic transport. Nature 525, 129-133 (2015).

159. P. Ciryam, G. G. Tartaglia, R. I. Morimoto, C. M. Dobson, M. Vendruscolo, Widespread aggregation and neurodegenerative diseases are associated with supersaturated proteins. Cell Rep. 5, 781-790 (2013).

160. A. B. Oromendia, A. Amon, Aneuploidy: implications for protein homeostasis and disease. Dis. Model. Mech. 7, 15-20 (2014).

161. D. M. Walther et al., Widespread Proteome Remodeling and Aggregation in Aging C. elegans. Cell 161, 919-932 (2015).

162. E. Cohen, J. Bieschke, R. M. Perciavalle, J. W. Kelly, A. Dillin, Opposing activities protect against age-onset proteotoxicity. Science 313, 1604-1610 (2006).

163. D. Kaganovich, R. Kopito, J. Frydman, Misfolded proteins partition between two distinct quality control compartments. Nature 454, 1088-1095 (2008).

164. S. F. Falsone et al., SERF protein is a direct modifier of amyloid fiber assembly. Cell Rep. 2, 358-371 (2012).

165. S. Escusa-Toret, W. I. Vonk, J. Frydman, Spatial sequestration of misfolded proteins by a dynamic chaperone pathway enhances cellular fitness during stress. Nat. Cell Biol. 15, 1231-1243 (2013).

166. S. M. Hill, X. Hao, B. Liu, T. Nystrom, Life-span extension by a metacaspase in the yeast Saccharomyces cerevisiae. Science 344, 1389-1392 (2014).

167. E. M. Sontag, W. I. M. Vonk, J. Frydman, Sorting out the trash: The spatial nature of eukaryotic protein quality control. Curr. Opin. Cell Biol. 26, 139-146 (2014).

168. E. W. J. Wallace et al., Reversible, specific, active aggregates of endogenous proteins assemble upon heat stress. Cell 162, 1286-1298 (2015). 
169. M. Arrasate, S. Mitra, E. S. Schweitzer, M. R. Segal, S. Finkbeiner, Inclusion body formation reduces levels of mutant huntingtin and the risk of neuronal death. Nature 431, 805-810 (2004).

170. E. T. Powers, R. I. Morimoto, A. Dillin, J. W. Kelly, W. E. Balch, Biological and chemical approaches to diseases of proteostasis deficiency. Annu. Rev. Biochem. 78, 959991 (2009).

171. Y. Miyata, H. Nakamoto, L. Neckers, The therapeutic target Hsp90 and cancer hallmarks. Curr. Pharm. Design 19, 347-365 (2013).

172. X. Li, H. Shao, I. R. Taylor, J. E. Gestwicki, Targeting allosteric control mechanisms in heat shock protein 70 (Hsp70). Curr. Top. Med. Chem. PMID: 27072701 (2016).

173. P. Tsaytler, H. P. Harding, D. Ron, A. Bertolotti, Selective inhibition of a regulatory subunit of protein phosphatase 1 restores proteostasis. Science 332, 91-94 (2011).

174. I. Das et al., Preventing proteostasis diseases by selective inhibition of a phosphatase regulatory subunit. Science 348, 239-242 (2015).

175. J. Leitman et al., ER stress-induced eIF2- $\alpha$ phosphorylation underlies sensitivity of striatal neurons to pathogenic huntingtin. PLoS One 9, e90803 (2014).

176. C. Sidrauski et al., Pharmacological brake-release of mRNA translation enhances cognitive memory. eLife 2, e00498 (2013).

177. Y. Sekine et al., Stress responses. Mutations in a translation initiation factor identify the target of a memory-enhancing compound. Science 348, 1027-1030 (2015).

178. C. Sidrauski et al., Pharmacological dimerization and activation of the exchange factor eIF2B antagonizes the integrated stress response. eLife 4, e07314 (2015).

179. B. Calamini et al., Small-molecule proteostasis regulators for protein conformational diseases. Nat. Chem. Biol. 8, 185-196 (2011).

180. C. B. Cooley et al., Unfolded protein response activation reduces secretion and extracellular aggregation of amyloidogenic immunoglobulin light chain. Proc. Natl. Acad. Sci. U.S.A. 111, 13046-13051 (2014).

181. A. S. Mendez et al., Endoplasmic reticulum stress-independent activation of unfolded protein response kinases by a small molecule ATP-mimic. eLife 4, e05434 (2015).

182. V. Kakkar et al., The S/T-rich motif in the DNAJB6 chaperone delays polyglutamine aggregation and the onset of disease in a mouse model. Mol. Cell DOI: 10.1016/ j.molcel.2016.03.017 (2016).

183. M. Nagy, W. A. Fenton, D. Li, K. Furtak, A. L. Horwich, Extended survival of misfolded G85R SOD1-linked ALS mice by transgenic expression of chaperone Hsp110. Proc. Natl. Acad. Sci. U.S.A. 113, 5424-5428 (2016).

184. B. H. Lee et al., Enhancement of proteasome activity by a small-molecule inhibitor of USP14. Nature 467, 179-184 (2010). 
185. D. C. Rubinsztein, C. F. Bento, V. Deretic, Therapeutic targeting of autophagy in neurodegenerative and infectious diseases. J. Exp. Med. 212, 979-990 (2015).

186. G. Veit et al., From CFTR biology toward combinatorial pharmacotherapy: expanded classification of cystic fibrosis mutations. Mol. Biol. Cell 27, 424-433 (2016).

187. A. Baranczak, J. W. Kelly, A current pharmacologic agent versus the promise of next generation therapeutics to ameliorate protein misfolding and/or aggregation diseases. Curr. Opin. Chem. Biol. 32, 10-21 (2016).

\section{Acknowledgements}

This review focuses mainly on original publications of the last 15 years. We apologize to all colleagues whose important work could not be cited due to space limitations. Work in the authors' laboratory was supported by an EMBO long-term fellowship to D.B. (ALTF 11162014), an ERC Advanced Grant (to F.U.H.), the Center for Integrated Protein Science Munich (CiPSM) and the Munich Cluster for Systems Neurology (SyNergy). M.H.-H. acknowledges

funding by the Minerva Foundation. We thank G. Jayaraj for bioinformatics analyses and members of the group for critically reading the manuscript. 


\section{Figure Legends}

Fig. 1. Increasing complexity of proteomes from bacteria to human. The total number of proteins, the numbers of proteins $>100$ aa and $<50$ aa in length are shown for the predicted proteomes of Escherichia coli, Saccharomyces cerevisiae and human. Note the increase in the number and fraction of proteins $>100$ aa and of large multidomain proteins from E. coli to $S$. cerevisiae to human. Such proteins tend to populate intermediate states during folding, resulting in increased aggregation propensity and chaperone dependence.

Fig. 2. Protein aggregation competes with folding. (A) During folding, protein molecules sample various conformations while traveling downhill on a potential free energy surface (green) towards the thermodynamically favorable native state. Kinetically trapped on- or off-pathway intermediates occupy low energy wells (folding intermediates and partially folded states). Molecular chaperones provide assistance to folding by lowering free energy barriers and preventing aberrant inter-molecular interactions (red), which can lead to various forms of aggregates (amorphous, oligomeric, fibrillar). Fibrillar (amyloid-like) aggregates may be thermodynamically most stable. (B) Molecular chaperones promote protein folding by a generic mechanism of kinetic partitioning of non-native states. Many chaperones use ATP binding and hydrolysis to switch between low- and high-affinity states for folding intermediates (unfolded, partially folded) exposing hydrophobic amino acid residues. Binding to chaperone blocks aggregation and reduces the concentration of aggregation-prone molecules. Release from chaperone allows folding (burial of hydrophobic residues). Efficient folding occurs when the rate constant of folding $\left(k_{\text {fold }}\right)$ is faster than re-binding of folding intermediate to chaperone $\left(k_{\text {on }}\right)$ and aggregation $\left(k_{\mathrm{agg}}\right)$ is slower than $k_{\mathrm{on}}$. Figures modified from (13). 
Fig. 3. Protein folding on the ribosome. The polypeptide exit tunnel of the large ribosomal subunit, as seen in a longitudinal cut through the bacterial ribosome, is magnified and shown schematically. The nascent polypeptide chain of a multidomain protein is modeled into the tunnel forming a folded N-terminal domain of $\sim 200$ aa immediately outside the ribosomal exit port. This co-translational folding process may be assisted by chaperones that dock onto the ribosome at the opening of the exit tunnel. Completion of folding is mediated by downstream chaperones which have no direct affinity for the ribosome. 1, 2 and 3 mark regions of the tunnel where structure formation of the nascent chain, including folding of domains $<50$ aa, has been observed. PTC, peptidyl transferase center.

Fig. 4. Chaperone pathways of protein folding in the cytosol. For $\sim 70 \%$ of bacterial (A) and eukaryotic proteins (B), the ribosome and associated factors (trigger factor, TF, in bacteria; NAC, nascent chain associated complex, and RAC, ribosome associated complex, in eukaryotes) provide folding assistance. Downstream of the ribosome, Hsp70 (DnaK in bacteria) is the hub of the chaperone network. Hsp70 cooperates with Hsp40s (DnaJ in bacteria) and nucleotide exchange factors (NEFs; GrpE in bacteria) to fold $\sim 20 \%$ of the proteome. The remaining $\sim 10 \%$ of the proteome is passed on to the chaperonins for folding, GroEL/ES in bacteria and TRiC in eukaryotes. In eukaryotes, some nascent proteins are transferred directly to TRiC by prefoldin (Pfd). This alternative pathway dominates in archaea, which lack Hsp70 proteins. Eukaryotes also employ the Hsp90 chaperone system to catalyze the activation of metastable proteins such as kinases and transcription factors. Clients are transferred to Hsp90 via the Hsp70 system and the cochaperone Hop. The bacterial Hsp90, HtpG, is thought to act more generally in folding and functions without known co-chaperones. 
Fig. 5. Hsp70 conformational cycle. (A) Structure of the bacterial Hsp70, DnaK. ATP binding and hydrolysis at the nucleotide binding domain (NBD) drives large conformational changes in Hsp70. ATP binding induces the open state of Hsp70 (left; PDB 4B9Q), in which the $\alpha$-helical lid of the substrate binding domain (SBD) is attached to the NBD. Upon hydrolysis of ATP to ADP, Hsp70 transitions from the open state with high on- and off-rates for peptide substrate to the closed state (right; PDB 2KHO), in which NBD and SBD are separated and the $\alpha$-helical lid is closed over the peptide binding cleft (low on- and off-rates for peptide substrate). Bound nucleotide is shown with adenosine in red and phosphate in blue. Bound peptide with the sequence NRLLLTG is shown in space filling representation. (B) Cycle of substrate binding and release. Non-native polypeptide substrate interacts first with Hsp40 and is delivered to ATPbound Hsp70 (open state). Interaction with Hsp40 triggers ATP hydrolysis on Hsp70, generating the closed state. ADP release catalyzed by nucleotide exchange factor (NEF) and re-binding of ATP trigger substrate release for folding or possible transfer to downstream chaperones. Hip in metazoans stabilizes the ADP-state, thereby delaying substrate release. Prolonged binding of non-native protein to Hsp70 may favor degradation. Figure modified from (13).

Fig. 6. The chaperonins. (A) Structure of the group I bacterial chaperonin system GroEL/GroES. The crystal structure of the asymmetrical GroEL:7ADP:GroES complex (PDB 1PFQ) is shown on the left in space-filling representation. One subunit in each ring is displayed in schematic representation with the equatorial domain in blue, the intermediate domain yellow and the apical domain red. The conformations of the GroEL subunit in the open state and in the GroES-bound, closed state are shown in ribbon-representation in the middle and on the right, 
respectively. (B) Reaction cycle of GroEL/GroES. Substrate protein binds as folding intermediate to the empty GroEL ring and is encapsulated by GroES in an ATP-dependent step. The protein is free to fold within the chaperonin nano-cage for the time required to hydrolyze ATP on each subunit of the heptameric GroEL ring. ATP binding to the opposite ring then triggers the release of folded protein and GroES, completing the cycle. Incompletely folded protein will re-bind following release. Figures modified from (13). (C) Structure of the eukaryotic chaperonin TRiC. The crystal structure of the hexadecameric TRiC complex with both rings in the closed conformation (PDB 4V94) is shown on the left in space-filling representation. One subunit in each ring is displayed in schematic representation with the domains color coded as above. The subunit conformation of the TRiC subunit in the open state (PDB 3KFK) and in the closed state (PDB 4V94) with the $\alpha$-helical extensions covering the ring opening are shown in ribbon-representation in the middle and on the right, respectively. ADP is bound in the equatorial domain in the closed state. (D) TRiC mechanism. The subunit orientation of the TRiC complex is shown schematically as a top view of the ring. Non-native substrate protein binds in a distinct topology contacting the apical domains of specific subunits (left). Upon ATP binding, TRiC releases the protein in a sequential manner aided by asymmetry in ATP affinities of the TRiC subunits (middle). Following release, the protein folds to completion inside the TRiC cage, the hemispheres of which are partitioned into acidic and basic character.

Fig. 7. Hsp90 conformational cycle. (A) Crystal structures of the open state of Hsp90 from bacteria (PDB 2IOQ) and the closed state from yeast (PDB 2CG9). Each subunit of the Hsp90 homodimer consists of an N-terminal nucleotide binding domain (NTD), a middle domain and a C-terminal dimerization domain (CTD). (B) Hsp90 reaction cycle. Inactive substrate protein 
binds to the Hsp90 dimer. ATP binding shifts the Hsp90 conformational equilibrium to the closed state with the NTDs dimerized. This metastable state is committed to ATP hydrolysis, resulting in further conformational changes to the closed, twisted state. Release of ADP and $\mathrm{P}_{\mathrm{i}}$ returns Hsp90 to the open state. The bound client protein is conformationally activated as Hsp90 proceeds through its cycle. Cofactors Hop and Cdc37 delay the ATP hydrolysis step of the cycle. Aha1 stabilizes NTD dimerization, thereby accelerating ATP hydrolysis. p23 stabilizes the closed dimer, slowing the release of substrate protein from Hsp90.

Fig. 8. The proteostasis network. (A) Cells employ an extensive network of molecular chaperones and other factors to maintain proteome integrity. Specific chaperone components are listed that participate in promoting the folding of newly-synthesized proteins and destabilize nonnative protein conformations, as well as cooperate with the degradation machineries (the ubiquitin proteasome system [UPS] and autophagy/lysosomal degradation). Pathways promoted by chaperones are shown with green arrows, off-pathway reactions with red arrows. Various options to modulate the PN pharmacologically are also indicated. RQC, ribosome quality control machinery. (B) Balanced versus imbalanced PN. Protein misfolding and aggregate formation is shown as the key process that puts pressure on the PN, resulting in possible destabilization. Green circle: The PN at full capacity copes with the load of misfolded proteins by either refolding/disaggregating or degrading aberrant proteins. Red circle: The PN is progressively overwhelmed through a positive feedback loop in which protein misfolding and aggregation leads to aberrant interactions with key proteins (including limiting PN components), resulting in proteostasis impairment and eventual collapse. This downhill spiral is accelerated by the agedependent decline of proteostasis capacity and/or excessive production of aberrant proteins. 
Fig. 9. The subset of chaperones that belong to the core fitness genes of transformed human cells. (A) Venn diagram showing the overlap between the human chaperome and the core fitness genes (the essentialome) of K562 leukemia cells. Data from references $(123,127)$. (B) The chaperome (332) proteins divided into the major chaperone families. The number and fraction of essential chaperones (55 in total) are indicated for each family. TPR, tetratricopeptide repeat cochaperones. Note that the chaperonins contain the highest fraction of essential components, and the TPR proteins comprise the highest number of essential cochaperones. Data from references $(123,127)$. 

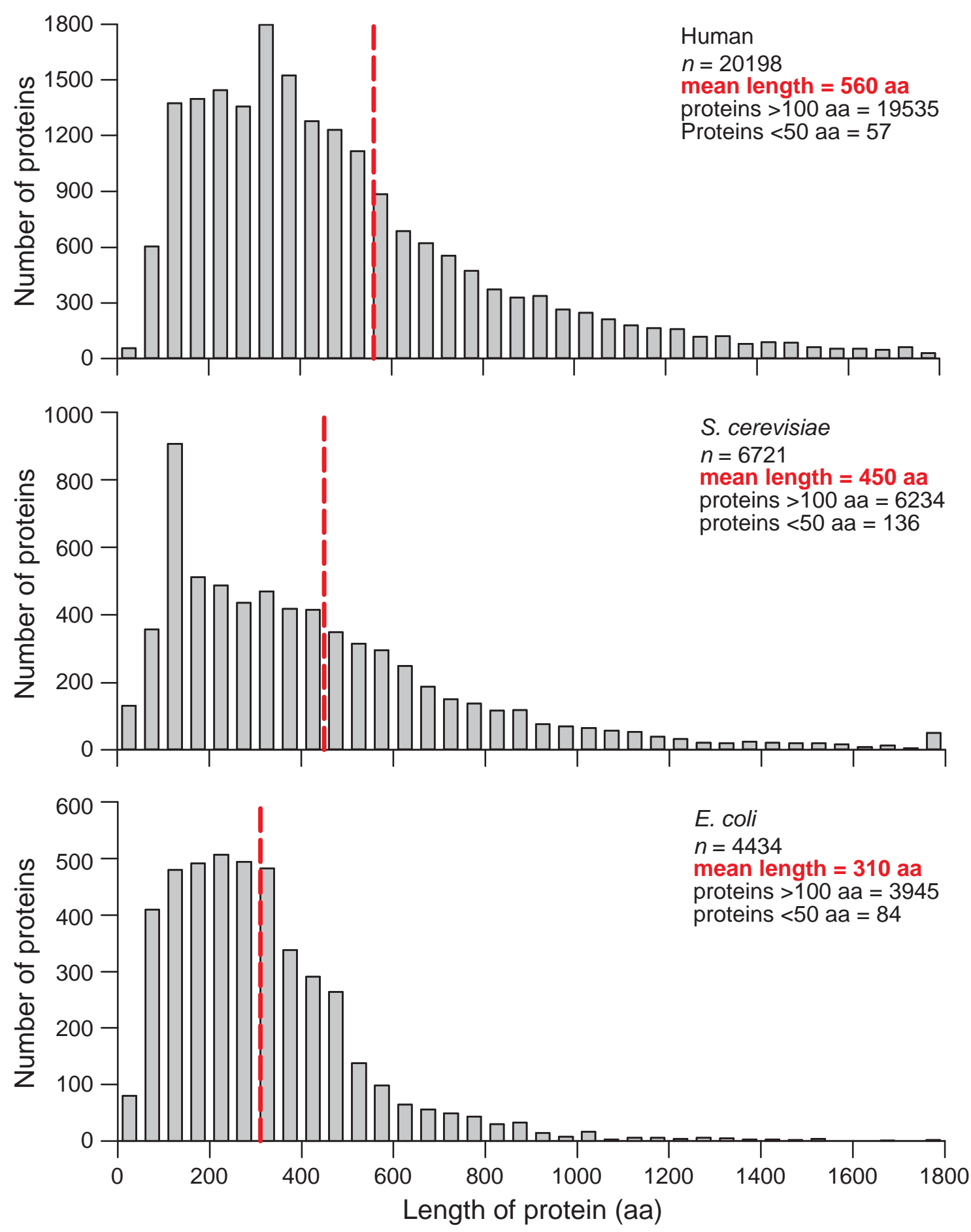


\section{Balchin et al., Figure 2}

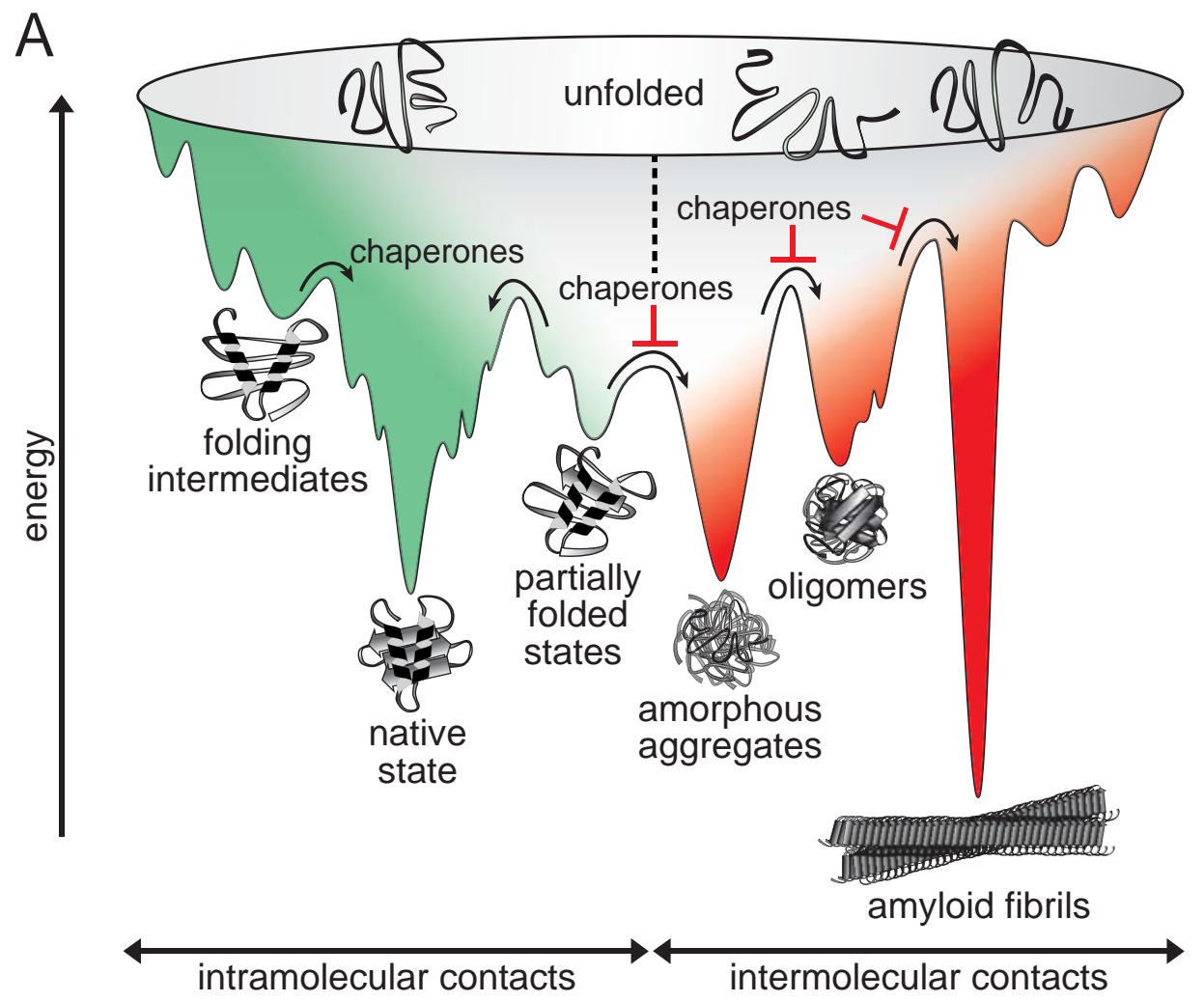

B

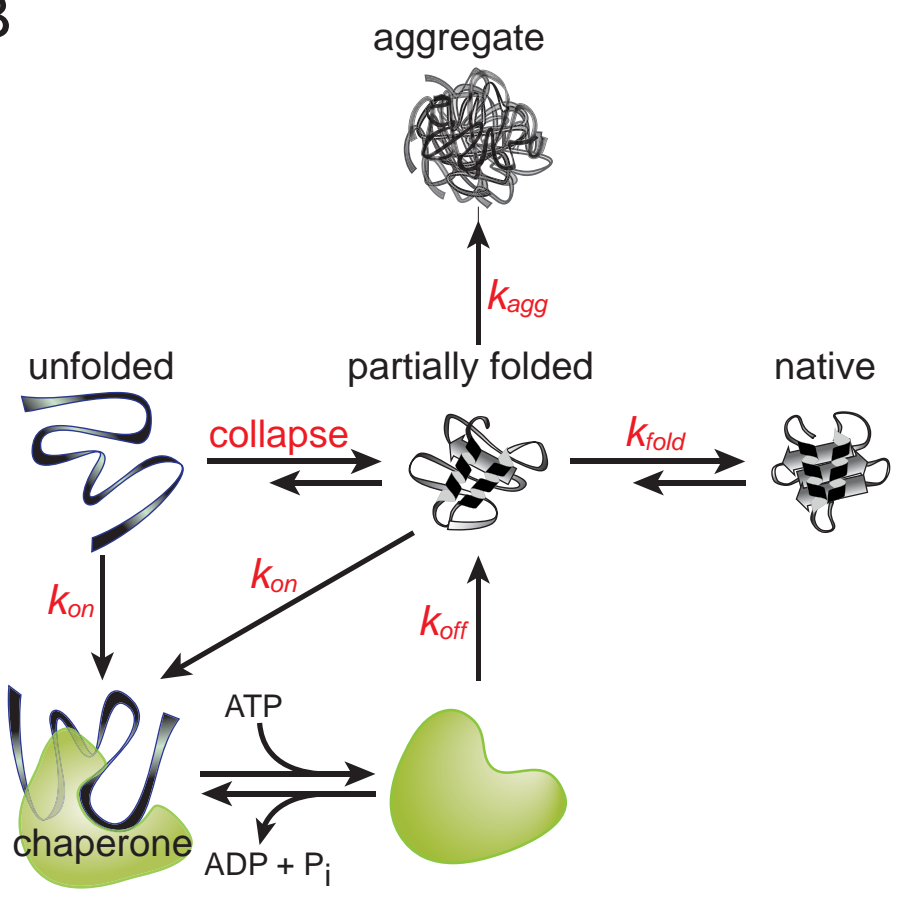




\section{Balchin et al., Figure 3}
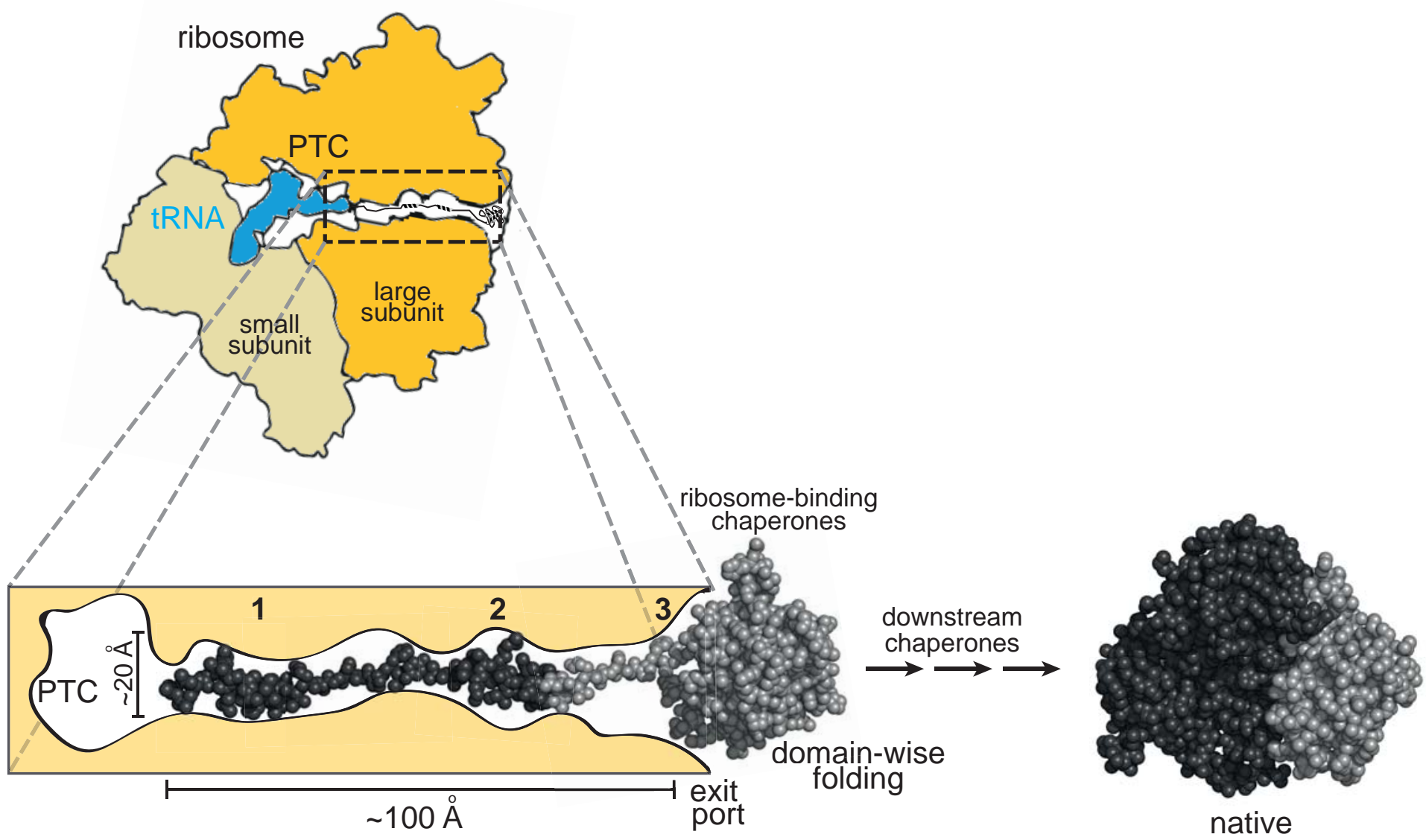


\section{Balchin et al., Figure 4}
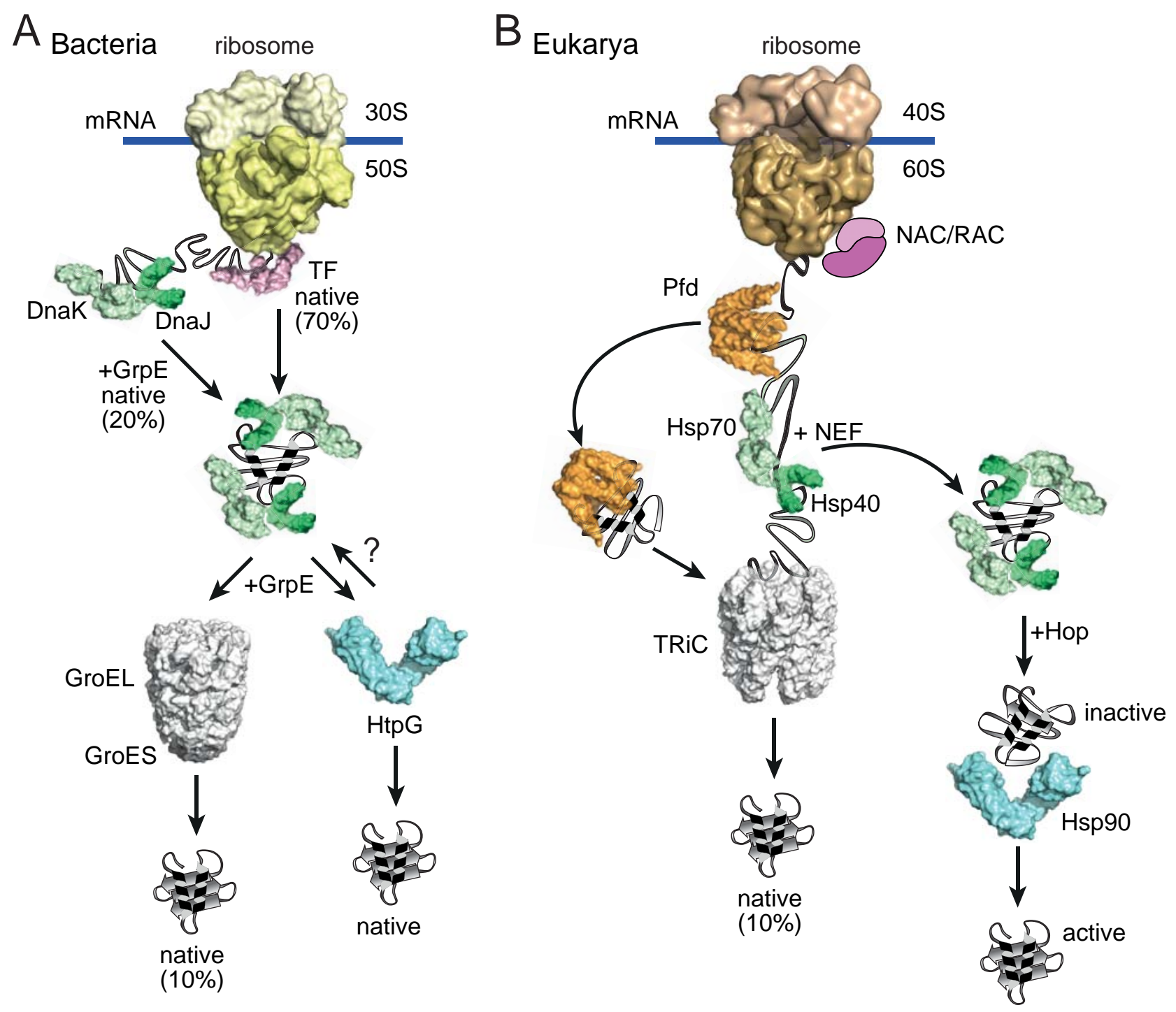


\section{Balchin et al., Figure 5}

A open state:

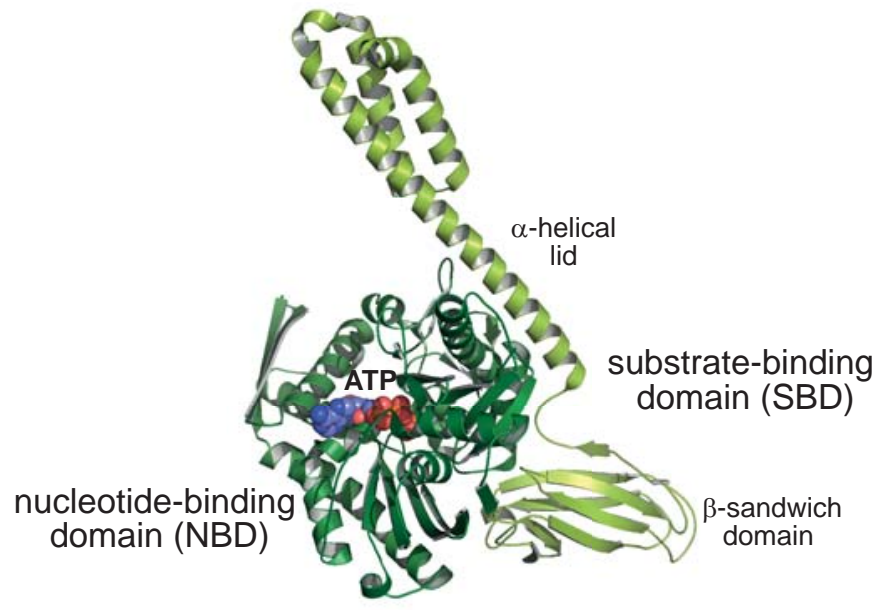

closed state:

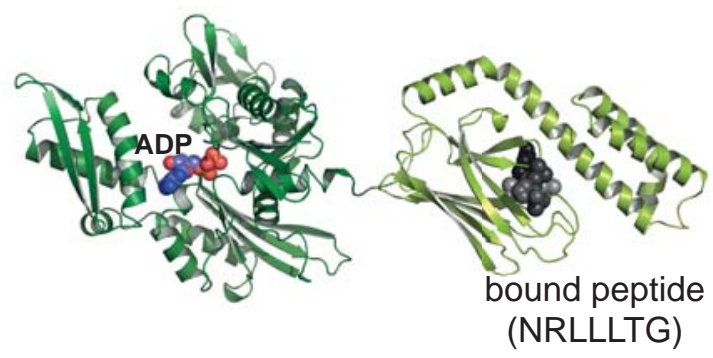

B

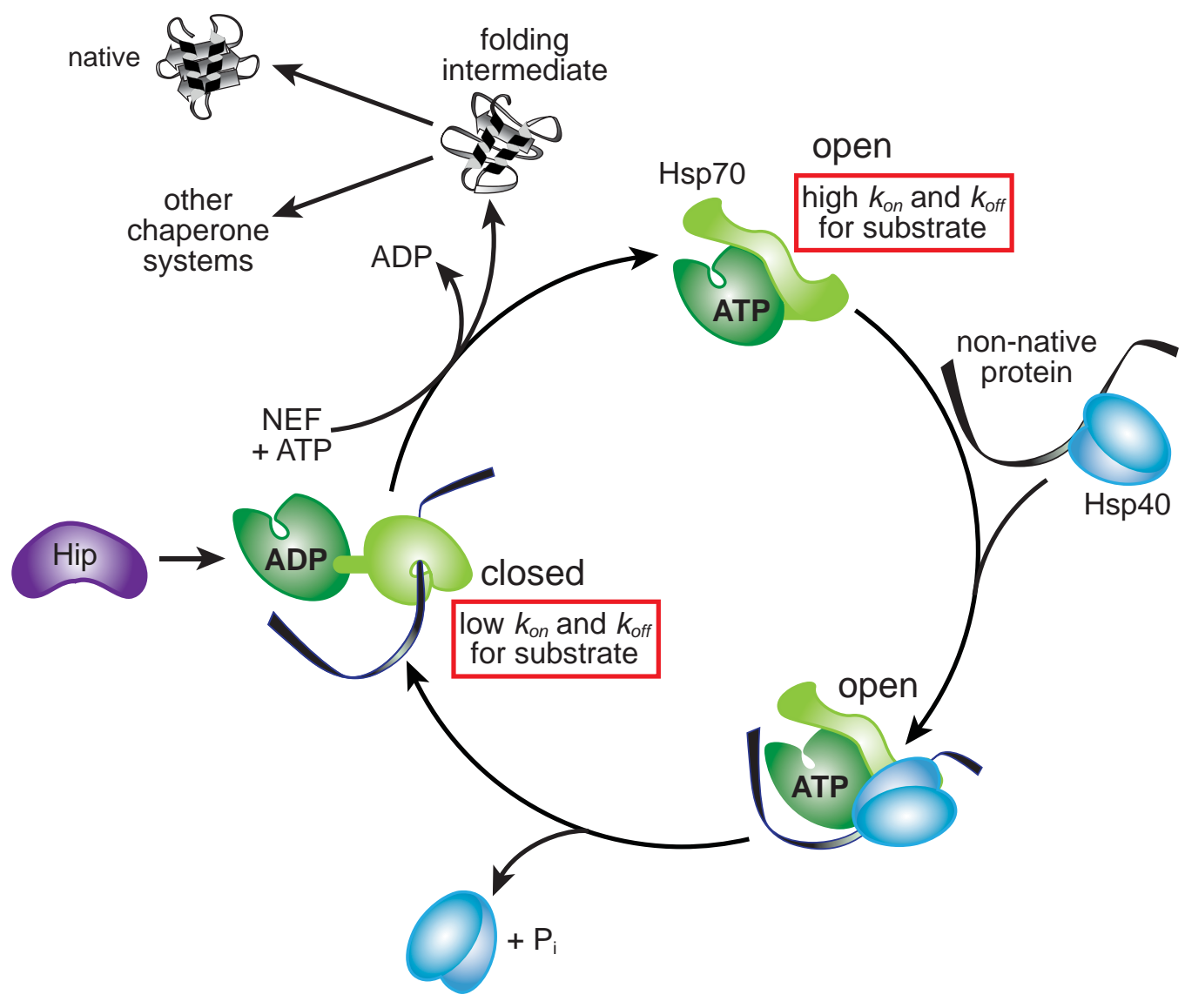




\section{Balchin et al., Figure 6}

A GroEL/GroES-ADP complex

open state:

closed state:
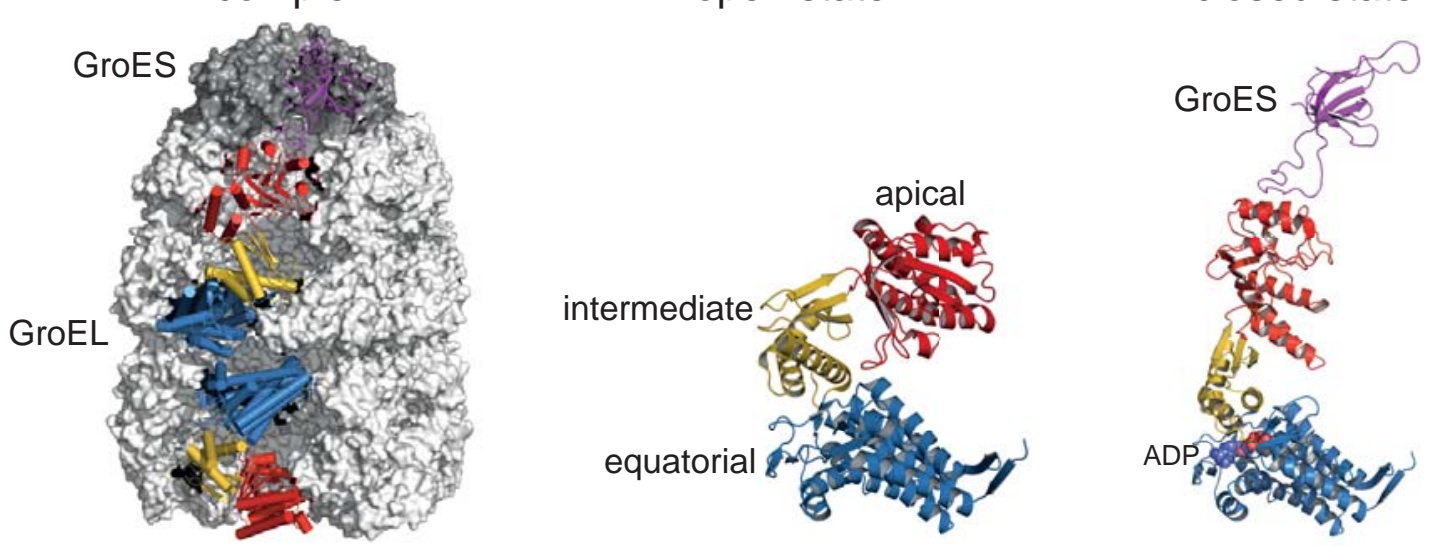

B

B folding

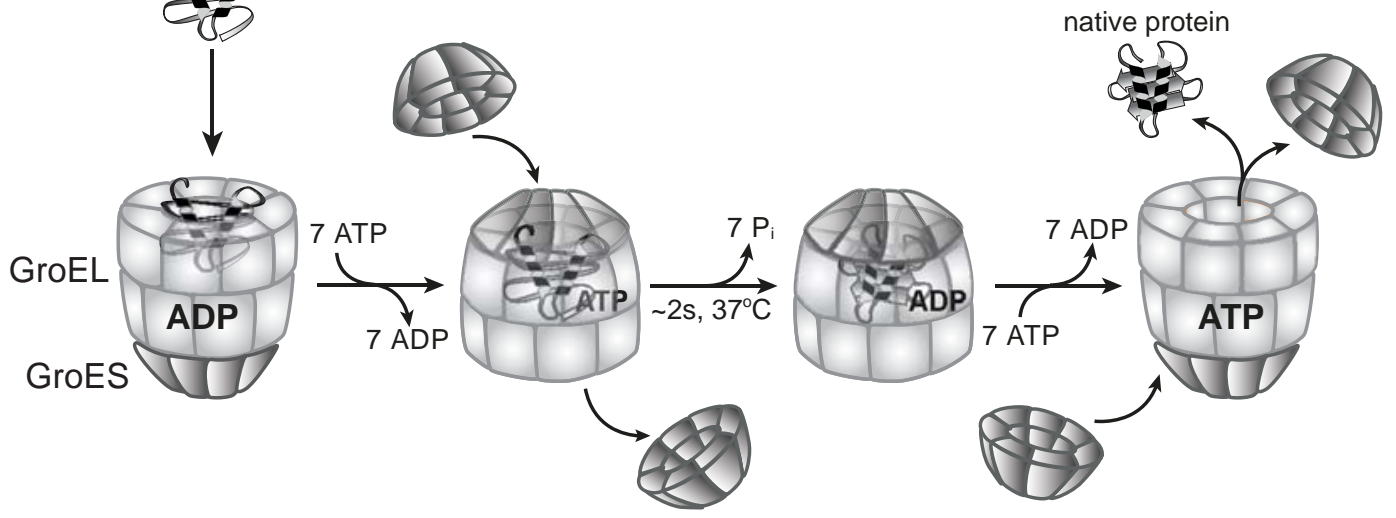

C

TRiC

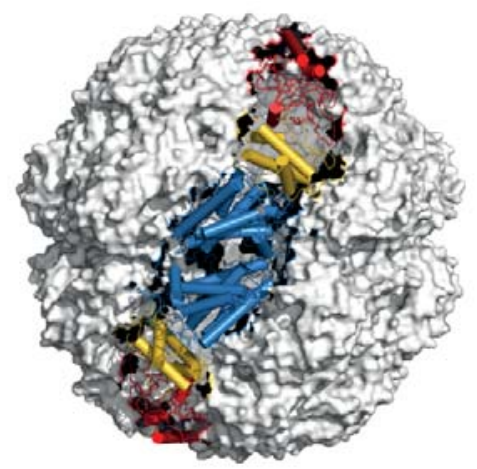

open state:

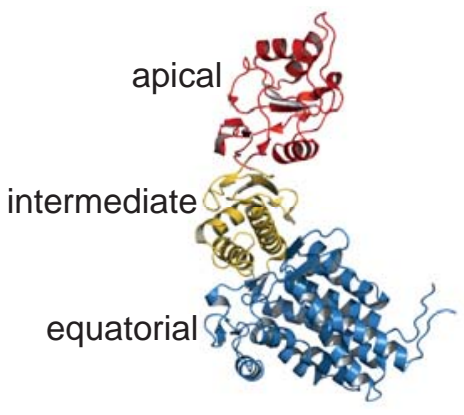

closed state:

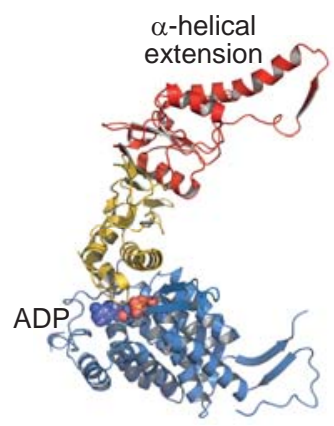

D substrate binding

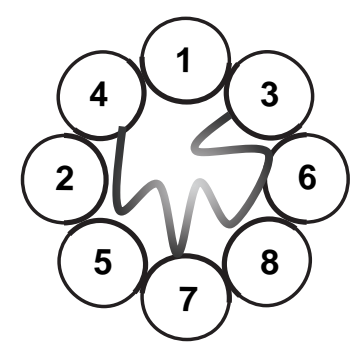

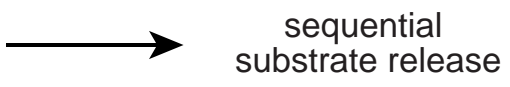

ATP binding folding in polarized chamber ATP hydrolysis
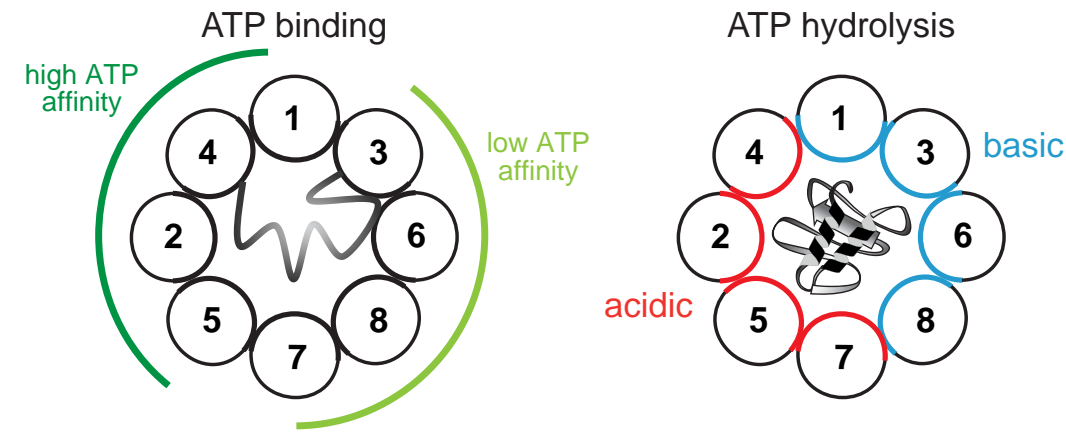


\section{Balchin et al., Figure 7}

A open state:

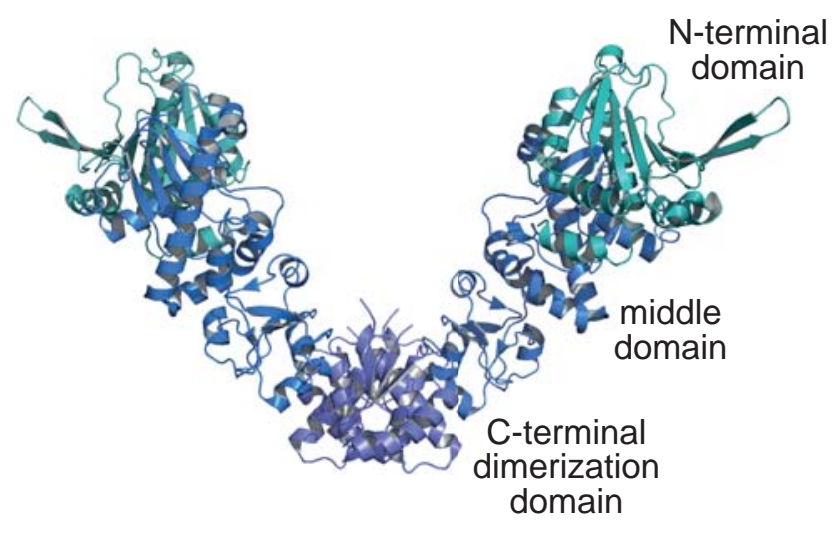

closed state:

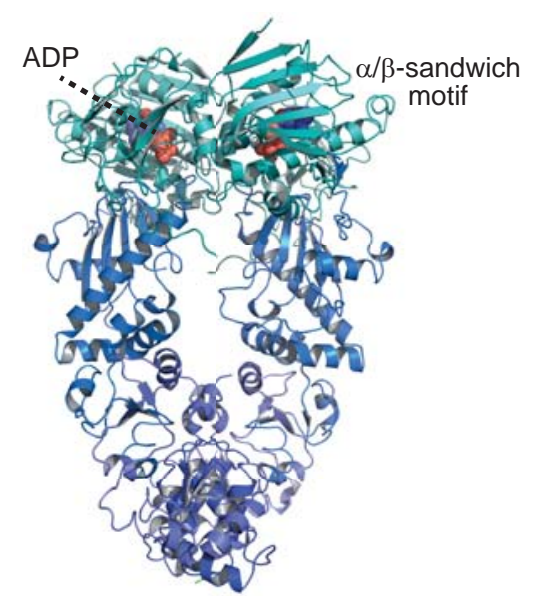

B
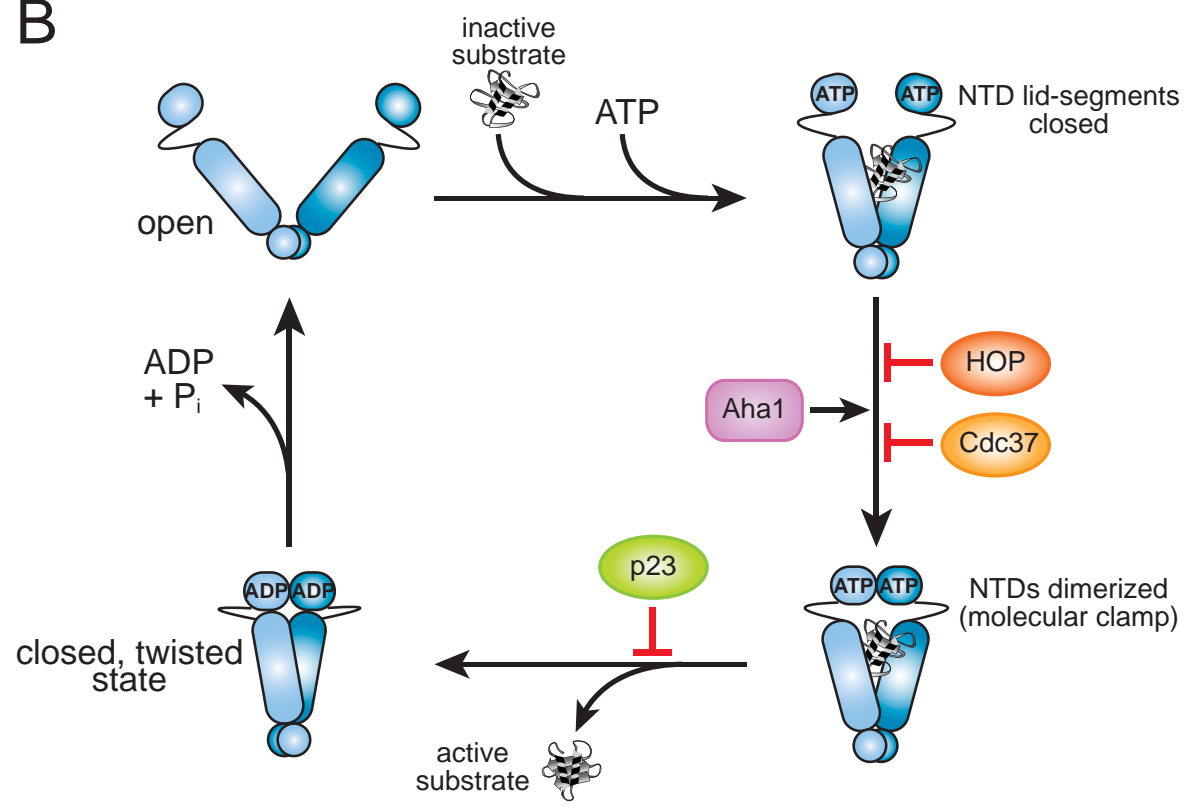


\section{Balchin et al., Figure 8}
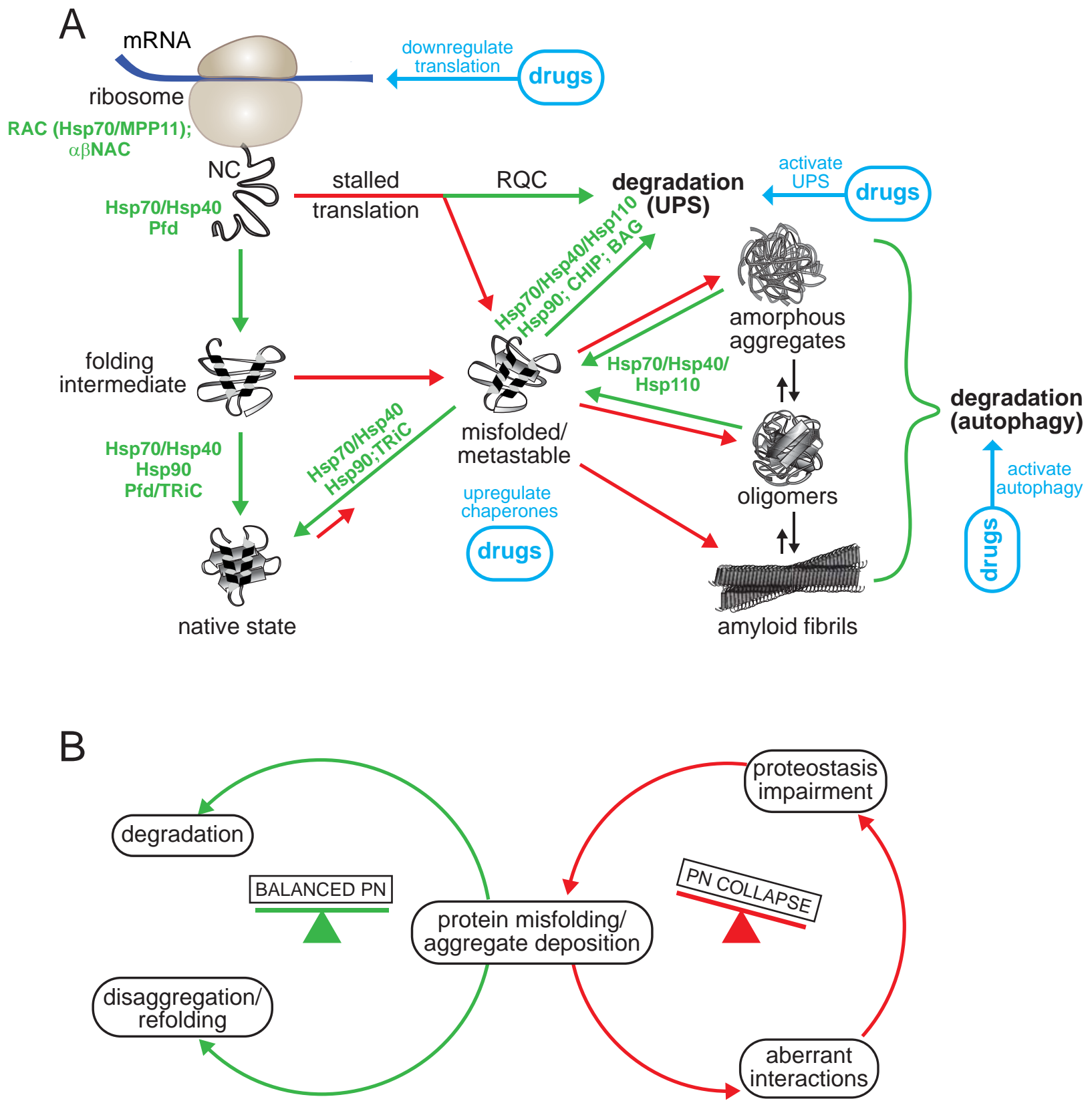
Balchin et al., Figure 9
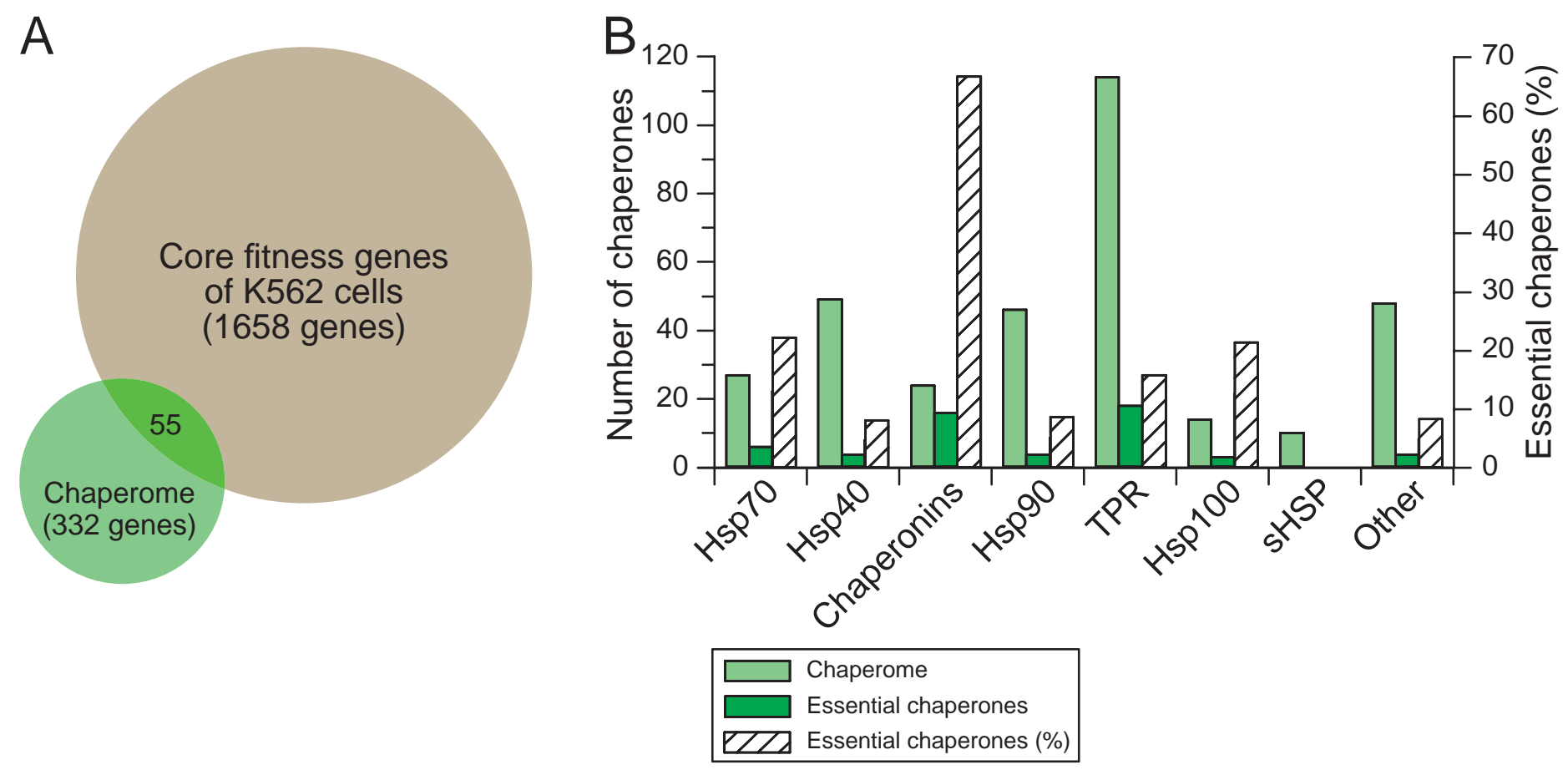